\title{
Carbodesilylierung von (Trimethylsilyl)imidazolen und -pyrazolen ${ }^{1)}$
}

\author{
Franz Effenberger*, Michael $\operatorname{Roos}^{2 a)}$, Roshan Ahmad ${ }^{2 b, c)}$ und Andreas Krebs \\ Institut für Organische Chemie der Universität Stuttgart, \\ Pfaffenwaldring 55, W-7000 Stuttgart 80 \\ Eingegangen am 21. Dezember 1990 \\ Key Words: Imidazoles, (trimethylsilyl)-, carbodesilylation of / Pyrazoles, (trimethylsilyl)-, carbodesilylation of / \\ Carbodesilylation
}

\begin{abstract}
Carbodesilylation of (Trimethylsilyl)imidazoles and -pyrazoles ${ }^{1)}$
The preparation of the 1-methyl(trimethylsilyl) (TMS)-substituted imidazoles $3 a, 4 a, 8,9$, and 11 a by silylation of the corresponding metallated imidazoles is described. Carbodesilylation of 3 with aldehydes or carboxylic halogenides occurs selectively in 2-position. In the presence of a strong base $(\mathrm{CsF})$ the reactivity against carbon electrophiles correlates well with the stability of the imidazolyl anions; regioselective

substituted imidazoles $\mathbf{3 a}$ and $\mathbf{9}$ therefore is possible, which allows the synthesis of a great variety of hydroxyalkyl-substituted imidazoles and of acylimidazoles. By using the dimethylsulfamoyl substituent as an $N$-protecting group, the $N$ unsubstituted 5-benzoylimidazole (26) as well as the comparable 5-benzoyl-pyrazole ( $30 \mathrm{~b}$ ) and 5-(hydroxyphenylmethyl)pyrazole (30 a) are accessible.
\end{abstract} carbodesilylation in 2-, 5-, or 4-position of the twofold TMS-
Imidazole und Pyrazole werden in der Regel über Kondensationsreaktionen aus geeigneten $\alpha$-substituierten Carbonyl- bzw. aus 1,3-Dicarbonylverbindungen mit Amidinen, Harnstoffen bzw. Hydrazinen erhalten; Acyl-substituierte Imidazole und Pyrazole können auf diesem Wege nur in begrenztem Maße dargestellt werden ${ }^{3)}$. Die bei Aromaten übliche Friedel-Crafts-Acylierung zur Einführung von AcylGruppen ist bei Heteroaromaten mit basischem Azin-Stickstoff wegen der Komplexierung des Katalysators bzw. des Elektrophils am Azin-Stickstoff und der dadurch stark herabgesetzten Reaktivität gegenüber Elektrophilen nur in Ausnahmefällen und unter speziellen Bedingungen möglich $^{4}$. In Einzelfällen sind 4(5)-Acylimidazole über PhotoFries-Umlagerungen von $N$-Acylimidazolen zugänglich ${ }^{5)}$.

Allgemein anwendbare Wege zur Darstellung von 4- und 5-Acylimidazolen sind einmal die Addition metallorganischer Verbindungen an Imidazol-4(5)-carbaldehyde und zum andern die Umsetzung metallierter Imidazole mit Aldehyden; die in beiden Fällen primär gebildeten sekundären Alkohole werden anschließend oxidiert ${ }^{6-10}$. Bei den Reaktionen metallierter Heterocyclen können sich deren leicht ablaufende Folgereaktionen (Isomerisierung, Ringöffnung, Umlagerung u. ä.) nachteilig auswirken ${ }^{11}$.

Als Alternative zur Verwendung metallierter Heterocyclen haben sich entsprechende Trimethylsilyl(TMS)-substituierte Verbindungen erwiesen, die mit Kohlenstoff-Elektrophilen, wie z.B. Carbonylverbindungen, unter Carbodesilylierung ebenfalls zu den erwünschten $C$-Substitutionsprodukten führen ${ }^{12}$. Starke Basen können über einen primären Angriff am Silicium der TMS-Gruppe die $\mathrm{Si}-\mathrm{C}$ Bindung in TMS-substituierten Aryl- und Hetaryl-Verbindungen unter Bildung von Carbanionen spalten, und zwar umso leichter, je stabiler die entsprechenden Aryl- bzw. Hetaryl-Anionen sind ${ }^{13)}$. Am Beispiel der Reaktionen von 1-
Methyl(trimethylsilyl)pyrazolen ${ }^{14)}, 5$-(Trimethylsilyl)pyrimi$\operatorname{din}^{1)}$ und TMS-substituierten Pyridinen ${ }^{1)}$ mit Elektrophilen konnte die Möglichkeit der regioselektiven Halodesilylierung und Carbodesilylierung in Abhängigkeit von der Anionenstabilisierung und den Reaktionsbedingungen aufgezeigt werden.

In der vorliegenden Arbeit berichten wir über die Carbodesilylierung von TMS-substituierten Imidazolen, wobei wiederum der Regioselektivität und deren Beeinflussung durch die Reaktionsbedingungen besondere Bedeutung beigemessen wurde. Darüber hinaus haben wir uns mit der Frage geeigneter $N$-Schutzgruppen für die Reaktionen von (Trimethylsilyl)imidazolen und -pyrazolen beschäftigt.

\section{Darstellung TMS-substituierter 1-Methylimidazole}

Die Einführung der TMS-Gruppe in den Imidazolring erfolgt in der Regel über die Reaktion entsprechender metallorganischer Verbindungen mit Trimethylsilylchlorid ${ }^{15)}$, wobei die metallorganischen Verbindungen durch direkte Metallierung von 1-Methylimidazol (1 a) oder durch einen Metall-Halogen-Austausch entsprechender Halogen-substituierter 1-Methylimidazole erhalten werden können. In Schema 1 ist die Darstellung der von uns untersuchten TMS-substituierten 1-Methylimidazole zusammengefaßt.

Nach der in der Literatur beschriebenen Umsetzung von 1 a mit $n$-Butyllithium und Trimethylsilylchlorid (TMSC) in Ether ${ }^{15 a)}$ erhielten wir in bescheidenen Ausbeuten ein Gemisch der mono- und disilylierten Verbindungen 2, 3a und 4a. In Gegenwart molarer Mengen 1,2-Bis(dimethylamino)ethan (TMEDA), über dessen günstigen Einfluß auf Reaktivität und Selektivität bei Lithiierungen vielfach berichtet wurde ${ }^{16)}$, isolierten wir dagegen ausschließlich 1-Methyl-2,5bis(trimethylsilyl)imidazol (3a) mit 68\% Ausbeute, das nach 
Lit. ${ }^{15 a)}$ in einer selektiv verlaufenden Hydrolyse mit Wasser bzw. Wasser/verd. Salzsäure zu 1-Methyl-5-(trimethylsilyl)imidazol (4a) protodesilyliert werden kann. Wir haben gefunden, daß sich für diese Hydrolyse Methanol besonders gut eignet und $4 \mathbf{a}$ auf diese Weise mit $85 \%$ Ausbeute erhalten wird.

Schema 1
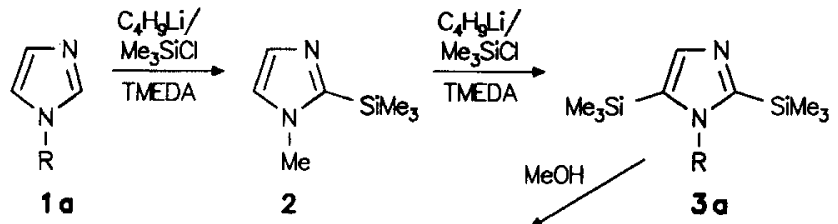

$\mid \begin{aligned} & \mathrm{Br}_{2} / \mathrm{AcONa} \\ & \text { Eisessig }\end{aligned}$<smiles>[R]n1cncc1[SiH3]</smiles><smiles>Cn1cncc1Cl</smiles>

40

5<smiles>Cn1c(Br)nc(Br)c1Br</smiles>

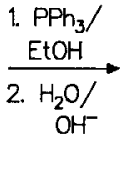

6<smiles>Cn1cnc(Br)c1Br</smiles><smiles>C[AsH2][TeH]</smiles><smiles>Cn1cnc(Br)c1[SiH3]</smiles>

$\mid \begin{aligned} & 1^{\prime} \mathrm{Mg} " / \mathrm{Me}_{3} \mathrm{SiCl} / \mathrm{THF} \\ & \text { 2. } \mathrm{H}_{2} \mathrm{O} / \mathrm{NaHCO}_{3}\end{aligned}$<smiles>C[SiH2]c1ncn(C)c1[SiH2]C</smiles><smiles>Cn1cnc(Cl)c1</smiles>
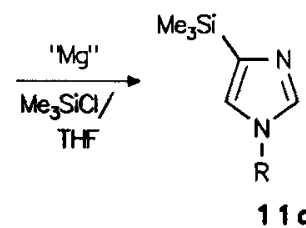

\begin{tabular}{r|cc}
$1,3,4,11$ & a & b \\
\hline $\mathrm{R}$ & $\mathrm{Me}$ & $\mathrm{SO}_{2} \mathrm{MMe}_{2}$
\end{tabular}

Die nur mit schlechter Ausbeute (15\%) beschriebene Darstellung von 2,4,5-Tribrom-1-methylimidazol (6) aus $1 \mathrm{a}^{17 \text { a) }}$ konnten wir unter den für die Bromierung von Imidazol angewandten Bedingungen ${ }^{17 b)}$ auf 33\% verbessern. Die Dehalogenierung von 6 erfolgte unter den Bedingungen der Protodebromierung von 2,4,5-Tribrom- $1 H$-imidazol ${ }^{18)}$ sehr selektiv und mit 80proz. Ausbeute zu 4,5-Dibrom-1-methylimidazol (7), aus dem über eine In-situ-Grignard-Reaktion mit $\mathrm{Mg} / \mathrm{TMSC}^{19)}$ bei Raumtemperatur 4-Brom-1-methyl-5(trimethylsilyl)imidazol (8) in 77 proz. Ausbeute erhalten wurde.

Ebenfalls über eine In-situ-Grignard-Reaktion gelang die Silylierung sowohl von 5-Chlor-1-methylimidazol (5) zu $\mathbf{4 a}$ als auch von 4-Chlor-1-methylimidazol (10) zu 1-Methyl-4(trimethylsilyl)imidazol (11 a) mit Mg/TMSC in Tetrahydrofuran (THF) nach mehrstündigem Erhitzen unter Rückfluß mit 81 bzw. $58 \%$ Ausbeute.

Versuche zur Darstellung von 1-Methyl-4,5-bis(trimethylsilyl)imidazol (9) aus der Dibromverbindung 7 waren weder mit einem Überschuß an Mg/TMSC noch mit der Entrainment-Methode ${ }^{19)}$ erfolgreich. Dagegen verlief die Überführung von 6 in 9 unter In-situ-Grignard-Bedingungen in THF bei tiefer Temperatur $\left(2-15^{\circ} \mathrm{C}\right)$ und anschlieBender Aufarbeitung mit Wasser/Natriumhydrogencarbonat in sehr guten Ausbeuten.

\section{Reaktionen TMS-substituierter 1-Methylimidazole mit Kohlenstoff-Elektrophilen in Abhängigkeit von den Reaktionsbedingungen}

Vergleichbar zu den Befunden über Reaktionen TMSsubstituierter 1-Methylpyrazole ${ }^{14)}$ sollten sich auch TMSsubstituierte 1-Methylimidazole mit Elektrophilen - je nach den angewandten Reaktionsbedingungen - regioselektiv in den Positionen 2, 4 oder 5 des Imidazolrings umsetzen lassen. Die Auswahl der untersuchten und im vorhergehenden Abschnitt beschriebenen TMS-substituierten 1-Methylimidazole erfolgte so, daß die zu erwartende $\mathrm{Re}$ gioselektivität der elektrophilen Carbodesilylierung experi-

Schema 2
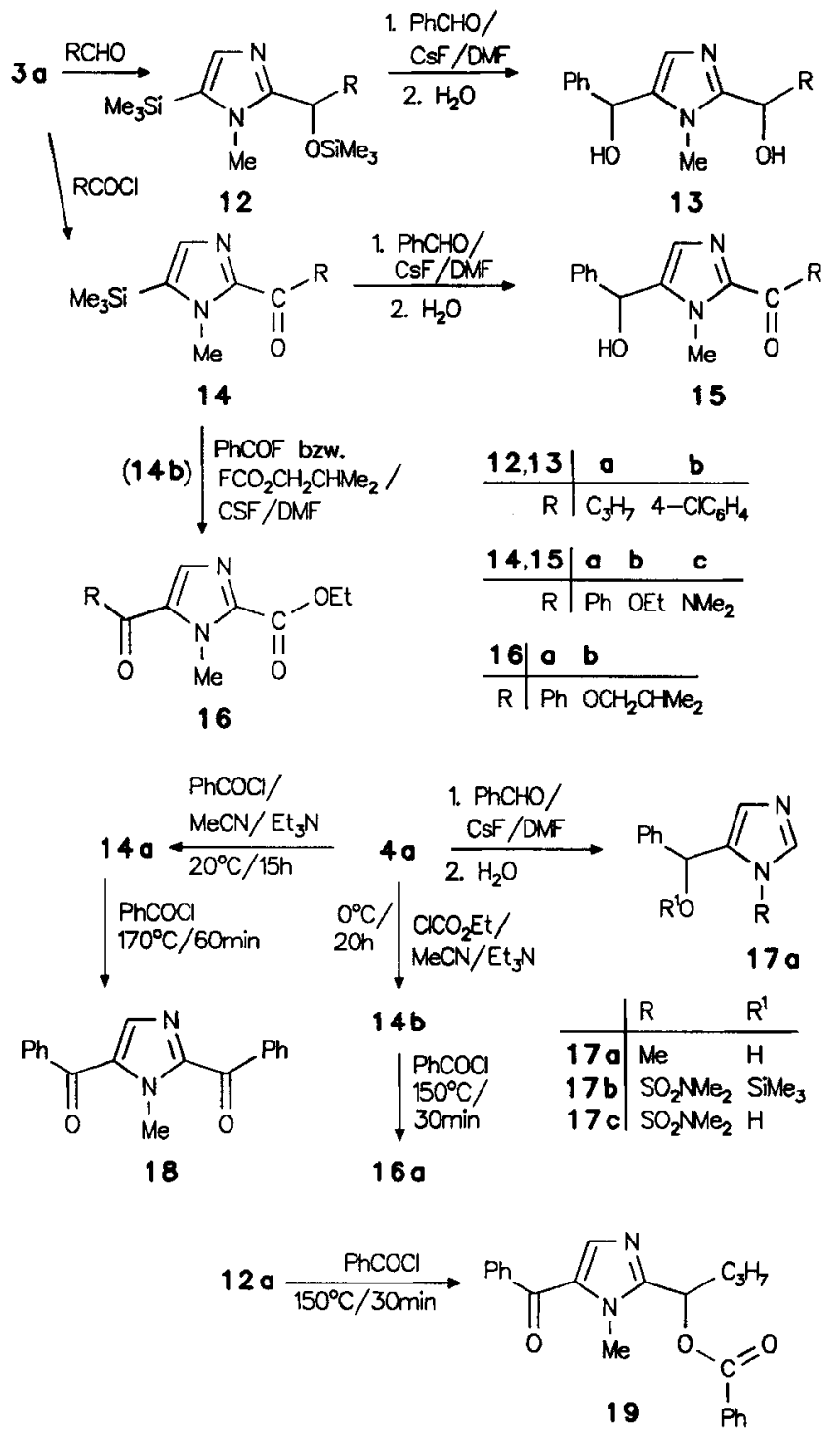
mentell belegbar wird, insbesondere auch durch die Reaktionen der 2,5- und 4,5-Bis(trimethylsilyl)-substituierten 1Methylimidazole 3a und 9.

Die Reaktivitätsabstufung in den Positionen 2, 4 und 5 sollte bei der direkten Umsetzung der TMS-substituierten 1-Methylimidazole mit Elektrophilen durch die relative Stabilität der Wheland-Zwischenstufen bestimmt sein, was eine Reaktivitätsreihenfolge $2 \gg 4 \approx 5$ ergeben würde ${ }^{20)}$; bei Zugabe starker Basen als Katalysator ${ }^{13,14)}$ sollte dagegen die relative Anionenstabilisierung in den verschiedenen Ringpositionen entscheidend sein, was zu einer Reaktivitätsreihenfolge $2>5>4^{9,21)}$ führen würde.

In Schema 2 und in den Tabellen 1 und 2 ist die Reaktionsweise der in 2- und 5-Position TMS-substituierten 1Methylimidazole 3a, 4a, 12 und 14 mit Kohlenstoffelektrophilen bei Temperaturen bis zu $100^{\circ} \mathrm{C}$ mit und ohne Zugabe von $\mathrm{CsF}$ als Katalysator zusammengefaßt.

1-Methyl-2,5-bis(trimethylsilyl)imidazol (3a) reagiert mit Aldehyden, Carbonsäurechloriden bzw. ChlorameisensäureDerivaten bei Temperaturen bis maximal $100^{\circ} \mathrm{C}$ ohne $\mathrm{CsF}$ Katalyse ausschließlich in 2-Position zu den Carbodesilylierungsprodukten 12 bzw. 14 (Schema 2, Tab. 1).

Tab. 1. Unkatalysierte Umsetzungen von 1-Methyl-2,5-bis(trimethylsilyl)imidazol (3a) mit Elektrophilen

\begin{tabular}{|c|c|c|c|c|}
\hline $\begin{array}{l}\text { Elektro- } \\
\text { phil }\end{array}$ & $\begin{array}{l}\text { Reakt } \\
\text { zeit } \\
h\end{array}$ & $\begin{array}{l}\text { ions- } \\
\text { temp. } \\
\text { oc }\end{array}$ & $\begin{array}{c}\text { Produkt } \\
-1-\text { methy } 1-5-(\text { tri- } \\
\text { methylsity } 1 \text { ) imidazol }\end{array}$ & $\begin{array}{l}\text { Ausb. } \\
(x)\end{array}$ \\
\hline $\mathrm{C}_{3} \mathrm{H}_{7} \mathrm{CHO}$ & 2 & 50 & $\begin{array}{l}2-[1-(\text { Trimethylsi loxy })- \\
\text { butyl]- (12a) }\end{array}$ & 86 \\
\hline 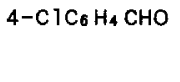 & 1 & 100 & $\begin{array}{l}2-[(4-\text { Ch lorpheny } 1)- \\
\text { (trimethylsi loxy })- \\
\text { methyl] }-(12 b)\end{array}$ & 90 \\
\hline $\begin{array}{l}\mathrm{C}_{6} \mathrm{H}_{5} \mathrm{COCl} \\
\mathrm{C} 1 \mathrm{CO} 2 \mathrm{Et} \\
\text { C } 1 \mathrm{CONMe2}\end{array}$ & $\begin{array}{c}1 / 2 \\
3 / 4 \\
3\end{array}$ & $\begin{array}{c}25 a) \\
40 a) \\
100\end{array}$ & $\begin{array}{l}\text { 2-Benzoyl- }(14 a) \\
2-(\text { Ethoxycarbonyl })-(14 b) \\
2-[(\text { Dimethylamino })- \\
\text { carbonyl }]-(14 c)\end{array}$ & b) $\begin{array}{l}97 \\
93 \\
47\end{array}$ \\
\hline
\end{tabular}

a) In $\mathrm{CCl}_{4}$.

Die Substitution der TMS-Gruppe in 5-Position der Imidazole 12, 14 und 4a durch Elektrophile erfolgt bei Temperaturen unterhalb $100^{\circ} \mathrm{C}$ in DMF nur in Gegenwart einer

Tab. 2. CsF-katalysierte Umsetzungen der 5-(Trimethylsilyl)-substituierten 1-Methylimidazole 4a, 12 und 14 mit Benzaldehyd (A), Benzoylfluorid (B) oder Fluorameisensäure-isobutylester (C) in DMF

\begin{tabular}{|c|c|c|c|c|c|}
\hline Edukt & $\begin{array}{l}\text { Elek- } \\
\text { tro- } \\
\text { phil }\end{array}$ & $\begin{array}{l}\text { Reakt } \\
\text { zeit } \\
\text { min }\end{array}$ & $\begin{array}{l}\text { tions- } \\
\text { temp. } \\
{ }^{\circ} \mathrm{C}\end{array}$ & $\begin{array}{l}\text { Produkt } \\
-5-(\text { hydroxypheny lmethy } 1)- \\
1-\text { methy } 1 \text { imidazo } 1\end{array}$ & $\begin{array}{r}\text { Ausb } \\
(x)\end{array}$ \\
\hline $\begin{array}{l}12 a \\
12 b\end{array}$ & A & $\begin{array}{l}120 \\
120\end{array}$ & $\begin{array}{l}90 \\
90\end{array}$ & $\begin{array}{l}\text { 2-(1-Hydroxybuty } 1)-(13 \mathbf{a}) \\
2-[(4-\text { Chlorpheny }])- \\
\text { hydroxymethy } 1]-(13 b)\end{array}$ & 43 \\
\hline $\begin{array}{l}14 a \\
14 b\end{array}$ & A & & $\begin{array}{l}45 \\
40\end{array}$ & $\begin{array}{l}\text { 2-Benzoyl- (15a) } \\
\text { 2-(Ethoxycarbony } 1)-(15 b)\end{array}$ & $\begin{array}{l}53 \\
58\end{array}$ \\
\hline $14 c$ & A & 225 & 30 & $\begin{array}{l}2-[(\text { Dimethy } 1 \text { amino })- \\
\text { carbony } 1]-(15 \mathrm{c})\end{array}$ & 46 \\
\hline $14 b$ & B & 40 & 60 & \multirow{3}{*}{\multicolumn{2}{|c|}{$\begin{array}{l}\text { 5-Benzoy 1-2-(ethoxycarbony } 1)- \\
1 \text {-methy } 1 \text { imidazo } 1 \text { (16a) } \\
\text { 2-(Ethoxycarbony } 1)-5-(\text { iso- } \\
\text { butoxycarbony } 1)-1 \text {-methy } 1- \\
\text { imidazol (16b) } \\
5-(\text { Hydroxypheny 1methy } 1)- \\
\text { 1-methylimidazo } 1 \text { (17a) }\end{array}$}} \\
\hline $14 b$ & $c$ & 150 & 50 & & \\
\hline $4 a$ & A & 120 & 90 & & \\
\hline
\end{tabular}

Base (z. B. CsF) zu den entsprechenden Carbodesilylierungsprodukten 13, 15, 16 und 17a (Schema 2, Tabellen 2, 3).

Tab. 3. Umsetzungen der 5-(Trimethylsilyl)-substituierten 1-Methylimidazole 4a, 12a und 14a,b mit Benzoylchlorid bzw. Chlorameisensäure-ethylester

\begin{tabular}{|c|c|c|c|c|c|}
\hline Edukt & $\begin{array}{l}\text { Elektro- } \\
\text { phil }\end{array}$ & $\begin{array}{c}\text { Reakt } \\
\text { zeit } \\
\text { h }\end{array}$ & $\begin{array}{l}\text { ions- } \\
\text { temp. } \\
\text { oc }\end{array}$ & $\begin{array}{c}\text { Produkt } \\
-1 \text {-methyl imidazol }\end{array}$ & $\begin{array}{l}\text { Ausb. } \\
(x)\end{array}$ \\
\hline $\begin{array}{r}4 a \\
4 a \\
12 a\end{array}$ & $\begin{array}{l}\text { PhCOCla) } \\
\text { ClCO2 Eta) } \\
\text { PhCOCl }\end{array}$ & $\begin{array}{r}15 \\
21 \\
1 / 2\end{array}$ & $\begin{array}{r}20 \\
0 \\
150\end{array}$ & $\begin{array}{l}14 a \\
14 b \mathbf{b}) \\
5-\text { Benzoy } 1-2-[1-(\text { ben- } \\
\text { zoy } 10 x y) \text { buty } 1]-(19)\end{array}$ & $\begin{array}{l}84 \\
57\end{array}$ \\
\hline $\begin{array}{l}14 a \\
14 b\end{array}$ & $\begin{array}{l}\mathrm{PhCOCl} \\
\mathrm{C} \mathrm{CO}_{2} \mathrm{Et}\end{array}$ & $\begin{array}{r}1 \\
1 / 2\end{array}$ & $\begin{array}{l}170 \\
150\end{array}$ & $\begin{array}{l}2,5-D i \text { benzoy } 1-(18) \\
16 a\end{array}$ & $\begin{array}{l}51 \\
59\end{array}$ \\
\hline
\end{tabular}

"In Gegenwart von Triethylamin in Acetonitril. - b) Außerdem $7 \% 4$ a.

Oberhalb von $100^{\circ} \mathrm{C}$ reagiert auch die TMS-Gruppe in 5-Position der Imidazole 12a und 14a,b mit Carbonsäurechloriden und Chlorameisensäureestern ohne Basenzusatz unter Carbodesilylierung zu den Disubstitutionsprodukten 19, 18 bzw. 16a (Schema 2, Tab. 3).

Eine Acylierung oder Carboxylierung in 2-Position ist auch ausgehend von in 2-Stellung nicht TMS-substituierten Imidazolen möglich, wie die Umsetzung von 1-Methyl-5(trimethylsilyl)imidazol (4a) mit Benzoylchlorid bzw. Chlorameisensäure-ethylester in Gegenwart von Triethylamin in Acetonitril zu 14a bzw. 14b zeigt (Schema 2, Tab. 3).

Einen Vergleich der Reaktivitäten der Positionen 2 und 4 zeigen die Umsetzungen von 1-Methyl-4-(trimethylsilyl)imidazol (11a) und 2-Benzoyl-1-methyl-4-(trimethylsilyl)imidazol (20a) mit Benzoylchlorid oder Chlorameisensäureethylester: Mit Benzoylchlorid reagiert 11 a erst bei höherer Temperatur $\left(140^{\circ} \mathrm{C}\right)$ unter ausschließlicher H-Substitution in 2-Position zu 20a, aus dem bei $170^{\circ} \mathrm{C}$ unter Carbodesilylierung der TMS-Gruppe in 4-Position 2,4-Dibenzoyl-1methylimidazol (21) entsteht. In Gegenwart von Triethylamin erfolgt dagegen schon bei $20^{\circ} \mathrm{C}$ in Acetonitril die $\mathrm{H}-$ Substitution in 2-Position von 11 a zu den 2-Acyl-1-methyl4-(trimethylsilyl)imidazolen 20a bzw. 20b (Schema 3, Tab. 4).

Tab. 4. Umsetzungen der 4-(Trimethylsilyl)-substituierten 1-Methylimidazole 11 a und 20a mit Benzoylchlorid sowie von 11 a mit Benzoylchlorid bzw. Chlorameisensäure-ethylester

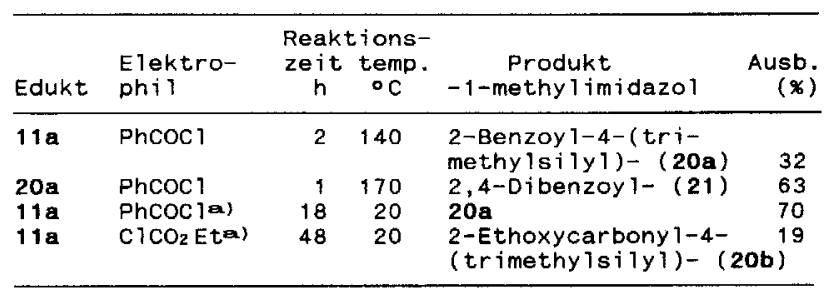

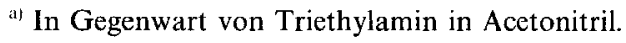

Die Reaktionsweise der 4,5-Bis(trimethylsilyl)-substituierten 1-Methylimidazole 9 und 23b mit einer Reihe von 
Elektrophilen unter Variation der Reaktionsbedingungen ist ebenfalls in Schema 3 sowie in Tab. 5 zusammengefaßt.

Schema 3

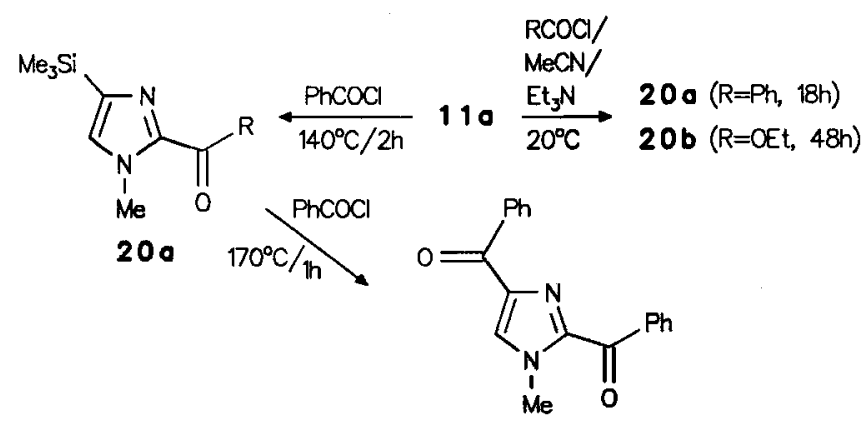

21

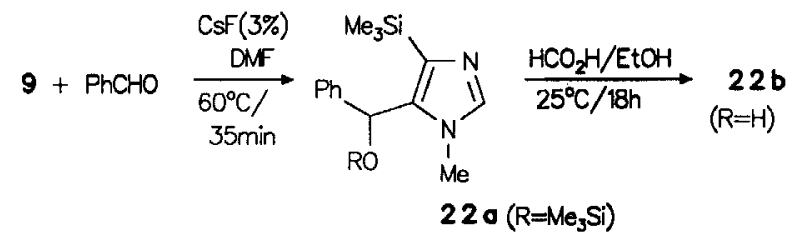

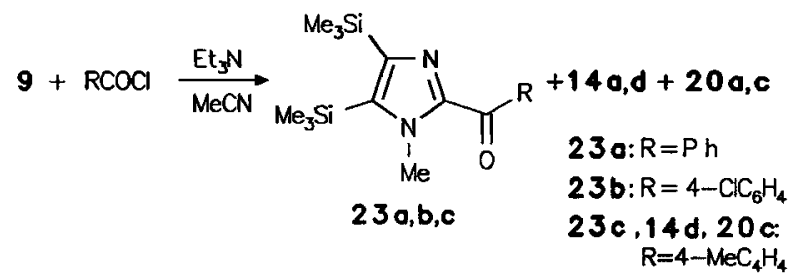

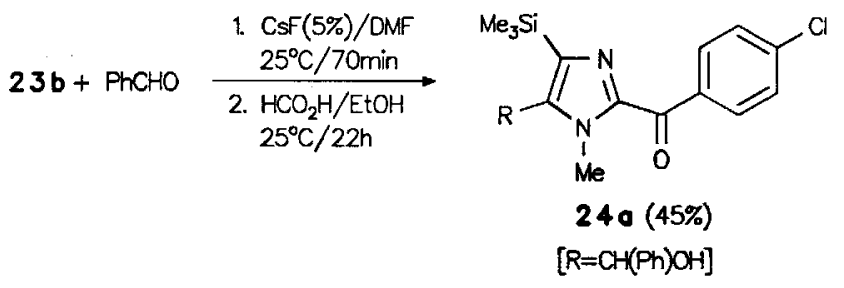

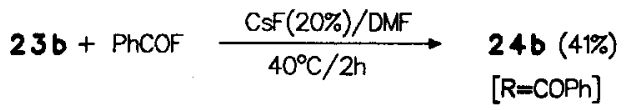

Mit CsF katalysiert, reagiert die TMS-Gruppe in 5-Position von 9 erwartungsgemäß deutlich besser als die in 4Position, so daß mit Benzaldehyd eine selektive Carbodesilylierung zu 22a möglich ist. Die Trimethylsiloxy-Gruppe in 22a kann mit Ethanol/Ameisensäure selektiv unter Bildung von 22 b hydrolysiert werden.

In Gegenwart von Triethylamin erfolgt mit Acylchloriden in Acetonitril eine Acylierung von 9 stets ausschließlich in 2-Position $\mathrm{zu}$ den 2-Acyl-1-methyl-4,5-bis(trimethylsilyl)imidazolen $23 \mathbf{a}, \mathbf{b}$, c, wobei unter den angewandten Reaktionsbedingungen zum Teil auch eine Protodesilylierung der TMS-Gruppe in 4-Stellung zu 14a, d bzw, in 5-Position zu 20 a, c eintritt.
Die Imidazole 23 reagieren mit Elektrophilen in Gegenwart von $\mathrm{CsF}$ bereits bei niederen Temperaturen $\left(25-40^{\circ} \mathrm{C}\right)$ in DMF ausschließlich nur in 5-Position unter Carbodesilylierung, wie am Beispiel der Umsetzungen von $23 \mathbf{b}$ mit Benzaldehyd und Benzoylfluorid zu 24a bzw. 24 b gezeigt wird.

Tab. 5. CsF-katalysierte Umsetzungen der 4,5-Bis(trimethylsilyl)(9) und -2-(4-chlorbenzoyl)-1-methylimidazole ( 23 b) mit Benzaldehyd und Aroylhalogeniden

\begin{tabular}{|c|c|c|c|c|}
\hline $\begin{array}{l}\text { Edukt/ } \\
\text { Elektropnt }\end{array}$ & $\begin{array}{l}\text { Reakt } \\
\text { zeit } \\
h\end{array}$ & $\begin{array}{l}\text { tions- } \\
\text { temp. } \\
\text { oc }\end{array}$ & $\begin{array}{c}\text { Produkt } \\
-1-\text { methylimidazol }\end{array}$ & $\begin{array}{l}\text { Ausb: } \\
\text { ( } \%)\end{array}$ \\
\hline 9/ $\mathrm{PhCHO}$ & $1 / 2$ & 60 & $\begin{array}{l}5-[\text { Pheny } 1 \text { (trimethylsil- } \\
\text { oxy)methy } 1]-4-(\text { trimethyl- } \\
\text { silyl)- (22a)a) }\end{array}$ & - \\
\hline 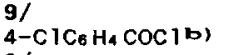 & 22 & 25 & $\begin{array}{l}4,5-\mathrm{Bis}\left(\text { trimethy } 1 \text { si } 1 y^{1}\right)- \\
2-(4-\mathrm{ch} \text { lorbenzoy } 1)-(23 \mathrm{~b})\end{array}$ & 69 \\
\hline 9/ 9 Phoc 7 b) & 22 & 50 & $\begin{array}{l}\text { 2-Benzoy } 1-4,5-\text { bis(tri- } \\
\text { methylsily } 1)-(23 a) \\
+20 a \\
+14 a\end{array}$ & $\begin{array}{r}7 \\
14\end{array}$ \\
\hline 9/ $\mathrm{CH}_{3} \mathrm{C}_{6} \mathrm{H}_{4} \mathrm{COC}\left(\mathrm{b}^{\circ}\right.$ & 80 & 25 & $\begin{array}{l}4,5-B \text { is (trimethylsilyl)- } \\
2-(4-\text { toluoyl)- (23c) } \\
+2-(4-\text { Toluoyl)-4-(tri- } \\
\text { methylsilyl)-(20c) } \\
+2-(4-\text { Toluoyl)-5-(tri- } \\
\text { methylsilyl)-(14d) }\end{array}$ & 32 \\
\hline $\begin{array}{l}\text { 23b/ } \\
\text { PhCHO }\end{array}$ & 1 & 25 & $\begin{array}{l}2-(4-\text { Ch lorbenzoy } 1)-5- \\
\text { [hydroxy (pheny } 1) \text { methy } 1]- \\
4-(\text { trimethy } 1 \text { si } 1 \text { y } 1)-(24 a)\end{array}$ & 45 \\
\hline $\begin{array}{l}\text { 23b/ } \\
\text { PhCOF }\end{array}$ & 2 & 40 & $\begin{array}{l}\text { 5-Benzoy } 1-2-(4-\text { chior- } \\
\text { benzoy } 1)-4-(\text { trimethy } 1- \\
\text { silyl)- (24b) }\end{array}$ & 41 \\
\hline
\end{tabular}

a) Nach 18stdg. Reaktion mit $\mathrm{HCO}_{2} \mathrm{H} / \mathrm{EtOH}$ bei Raumtemp. 64\% 5-(Hydroxyphenylmethyl)-1-methyl-4-(trimethylsilyl)-imidazol (22b). - b) Umsetzung ohne $\mathrm{CsF}$ in Gegenwart von Triethylamin in Acetonitril.

Durch Erhitzen der Diacylverbindung 24b mit Benzoylchlorid auf $170^{\circ} \mathrm{C}$ entstand eine Palette von Produkten, aus der sich chromatographisch eine siliciumfreie Verbindung abtrennen ließ, deren Molmasse dem 4,5-Dibenzoyl-2-(4chlorbenzoyl)-1-methylimidazol entspricht.

\section{Diskussion der Ergebnisse}

Vorstehend wurde gezeigt, daß eine selektive Acylierung oder Carboxylierung von TMS-substituierten 1-Methyl-imidazolen in den Positionen 2, 4 und 5 möglich ist. In den von uns untersuchten Carbodesilylierungsreaktionen von 1Methyl-2,5-bis(trimethylsilyl)imidazol (3a) war die TMSGruppe in 2-Position am reaktivsten, was sich bei den $\mathrm{Re}$ aktionen ohne Zugabe von Base (Schema 2) mit der gröBeren Stabilität der Wheland-Zwischenstufen ${ }^{20)}$ deuten läßt. In Gegenwart von Triethylamin ist die Acylierung in 2-Stellung sogar bevorzugt gegenüber einer Carbodesilylierung in 4- oder 5-Position (Schemata 2, 3). Es ist anzunehmen, daß diese Reaktionen über einen Ylidmechanismus ${ }^{1)}$ ablaufen, der durch eine konkurrierende Reaktion der Acylierungsagenzien mit dem Azin-Stickstoff der Imidazole einerseits und Triethylamin andererseits ermöglicht wird. Die Deprotonierung des Imidazoliumsalzes zu einem Ylid ist außerordentlich begünstigt ${ }^{22)}$ und dessen anschließende Acylierung leicht möglich ${ }^{1)}$, wie die in Schema 4 angeführte Acylierung von 9 beispielhaft zeigt. 
Schema 4

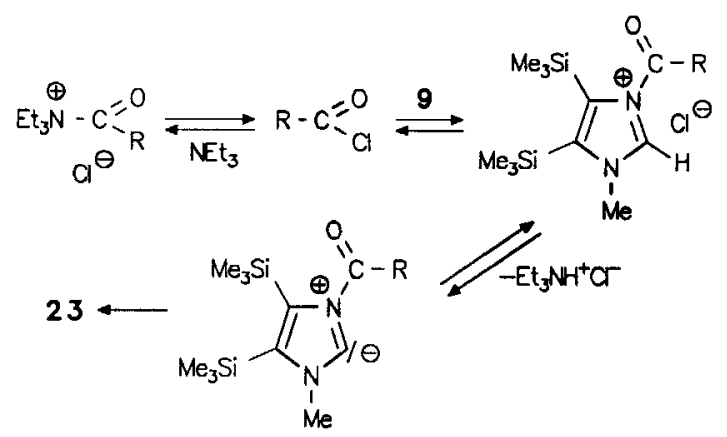

Carbodesilylierungen in 4- und in 5-Position, ohne $\mathrm{Zu}$ gabe von Base, sind nur bei Temperaturen $>150^{\circ} \mathrm{C}$ möglich. Da hierbei kein entscheidender Reaktionsunterschied beider Positionen zu erwarten war ${ }^{20}$, haben wir auf einen Reaktivitätsvergleich zwischen der 4- und 5-Position unter diesen Bedingungen verzichtet.

Völlig anders liegen die Verhältnisse der Carbodesilylierungen bei Zugabe starker Base (CsF), bei denen die Anionenstabilität der Zwischenstufen entscheidend ist ${ }^{1,14)}$. Bedingt durch den Adjacent-Lone-Pair-Effect ${ }^{23)}$, ist das 5-Imidazolyl-Anion deutlich stabiler als das 4-ImidazolylAnion ${ }^{421)}$. Damit wird eine selektive Substitution der TMSGruppe in 5-Stellung von 4,5-Bis(trimethylsilyl)-substituierten Imidazolen möglich, wie die Beispiele der Umsetzungen mit den Verbindungen 9 und 23b (Schema 3) belegen.

Zusammenfassend kann festgehalten werden, daß über TMS-substituierte 1-Methylimidazole durch geeignete Wahl der Reaktionsbedingungen die selektive Einführung von $\mathbf{H y -}$ droxyalkyl-, Acyl- und Carboxyl-Substituenten auf einfache Weise möglich ist.

\section{Darstellung und Reaktionen 1-(Dimethylsulfamoyl)- geschützter Imidazole und Pyrazole}

Imidazole und Pyrazole können aufgrund ihrer $\mathrm{N}-\mathrm{H}$ Acidität mit TMS-substituierten Imidazolen und Pyrazolen unter Protodesilylierung reagieren. Um diese störenden Nebenreaktionen zu vermeiden, haben wir sowohl bei den Reaktionen der Imidazole (vorliegende Arbeit) als auch bei den Umsetzungen der Pyrazole ${ }^{14)}$ jeweils die $N$-Methyl-Verbindungen eingesetzt. Da für die Herstellung der häufig erwünschten, am Stickstoff unsubstituierten Imidazole und Pyrazole eine Abspaltung der $N$-Methyl-Gruppe nicht möglich ist, mußte eine andere $N$-Schutzgruppe eingesetzt werden.

Die Einführung der TMS-Gruppe in Imidazole bzw. Pyrazole erfolgt häufig über Metallierungsreaktionen, was die Wahl der Schutzgruppe in starkem Maße einengt. Wir haben daher die Dimethylsulfamoyl-Schutzgruppe gewählt, die gegenüber Metallierungsagenzien weitgehend stabil ist und sich in saurem Medium einfach abspalten läßt ${ }^{8,24)}$.

Die Silylierung von 1-(Dimethylsulfamoyl)imidazol (1b) mit Butyllithium/TMSC in Dimethoxyethan ergibt in mäBigen Ausbeuten ein Gemisch aus den in 4- und den in 2und 4-Stellung TMS-substituierten Verbindungen $11 \mathrm{~b}$ und 25, aus dem bei wäßriger Aufarbeitung 1-(Dimethylsulfa- moyl)-4-(trimethylsilyl)imidazol (11 b) erhalten wird (Schema 5).

Bei der Silylierung von $\mathbf{1} \mathbf{b}$ mit Lithiumdiisopropylamid in Gegenwart von TMSC analog zur „In-situ“-GrignardReaktion ${ }^{19)}$ erfolgt ausschlieBlich Silylierung in 2- und 5Position zu einem Gemisch aus den Verbindungen $\mathbf{3 b}$ und 4b, aus dem man durch Aufarbeitung mit Methanol das 1(Dimethylsulfamoyl)-5-(trimethylsilyl)imidazol (4b) in 85 proz. Gesamtausbeute erhält.

Schema 5
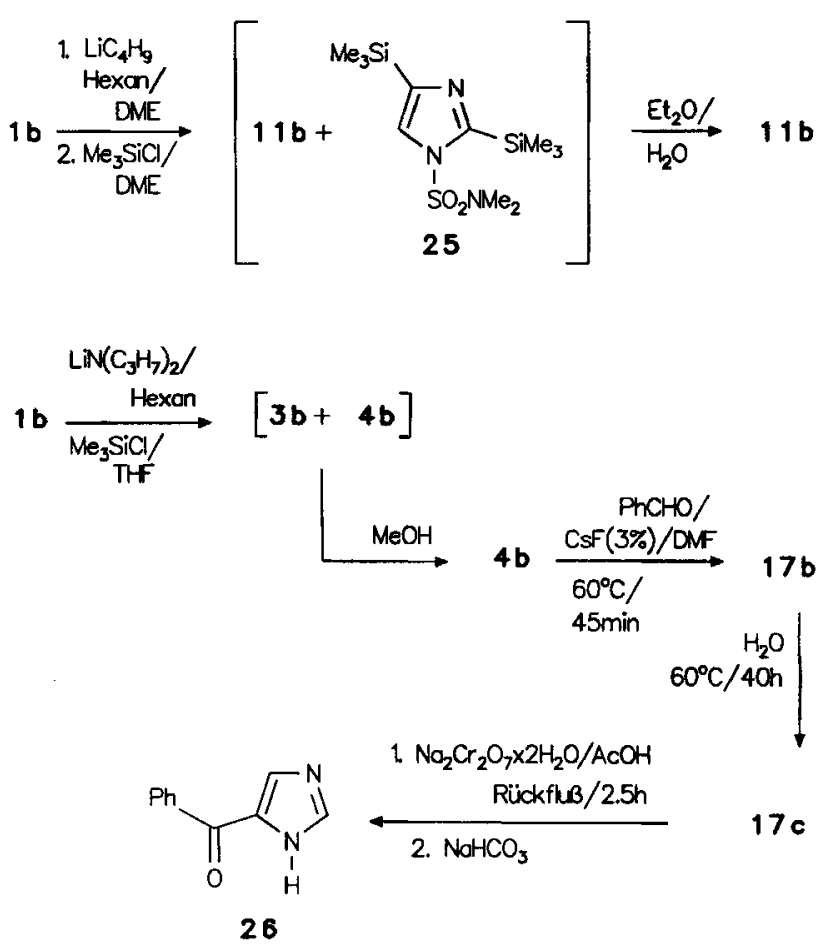

Die CsF-katalysierte Carbodesilylierung von $4 \mathrm{~b}$ mit Benzaldehyd zu $17 \mathrm{~b}$ gelang bereits bei $60^{\circ} \mathrm{C}$ in sehr guter Ausbeute. Für die vergleichbare Reaktion der $N$-Methylverbindung 4a mußten höhere Temperaturen und längere Reaktionszeiten angewendet werden (siehe oben), was durch die bessere Stabilisierung des 5-Imidazolyl-Anions in $\mathbf{1 b}$ im Vergleich zu 1 a erklärt werden kann. Durch Erwärmen mit Wasser kann der Silylether 17b zum Phenylcarbinol 17c gespalten werden, bei dessen Oxidation mit Natriumdichromat in Essigsäure überraschenderweise auch gleich die $N$ Schutzgruppe abgespalten wird und 5-Benzoylimidazol (26) als Endprodukt in befriedigender Ausbeute entsteht (Schema 5). Chadwick und Ngochindo ${ }^{8)}$ haben für die Abspaltung der Dimethylsulfamoyl-Schutzgruppe mehrstündiges Erhitzen in $2 \mathrm{~N} \mathrm{HCl}$ angegeben.

Als Ergänzung zu unseren früheren Untersuchungen ${ }^{14)}$ haben wir jetzt auch die Darstellung und das Reaktionsverhalten von 1-(Dimethylsulfamoyl)-5-(trimethylsilyl)pyrazol (28) untersucht.

Trotz der vergleichbaren $\mathrm{p} K_{\mathrm{s}}$-Werte von Imidazol und Pyrazol (27a) muß für die Sulfonylierung von 27a zu 1(Dimethylsulfamoyl)pyrazol (27 b) in Benzol unter Rückfluß 
Schema 6

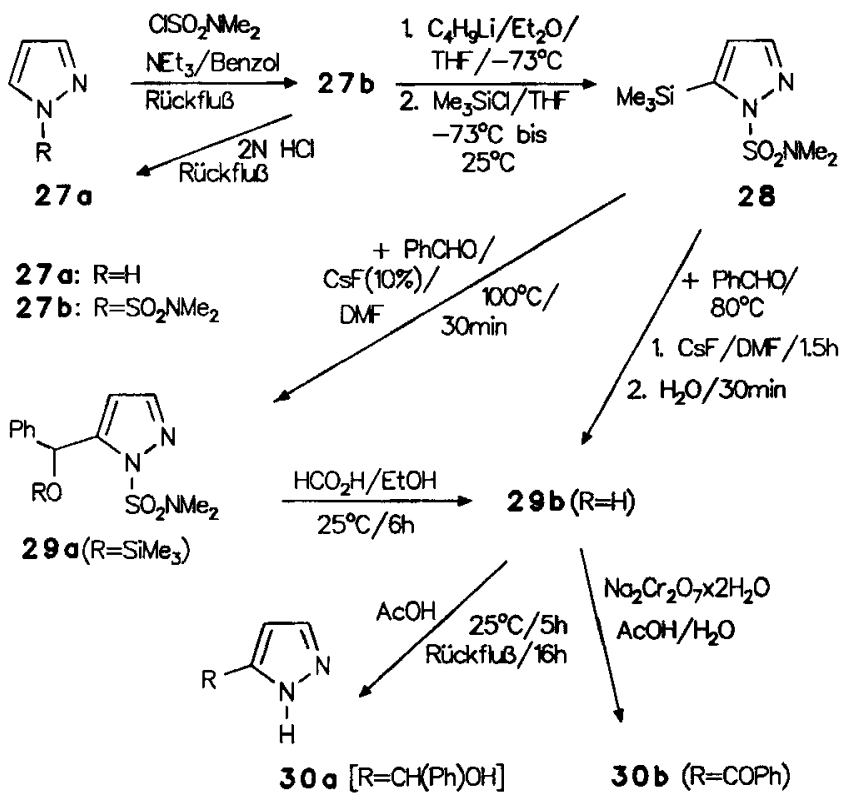

erhitzt werden, während Imidazol schon bei Raumtemperatur reagiert. Im Gegensatz zur Silylierung des 1-(Dimethylsulfamoyl)imidazols (1 b) kann das analog substituierte Pyrazol 27b erwartungsgemäß ohne Schwierigkeiten in 5Position metalliert und durch Umsetzung mit TMSC in sehr guten Ausbeuten in 1-(Dimethylsulfamoyl)-5-(trimethylsilyl)pyrazol (28) übergeführt werden (Schema 6). Die mit CsF katalysierte Carbodesilylierung von $\mathbf{2 8}$ mit Benzaldehyd führt nach kurzen Reaktionszeiten ausschließlich zum Substitutionsprodukt 29a, das mit Ameisensäure unter Silyletherspaltung das Carbinol 29b ergibt; bei längeren Reaktionszeiten und Aufarbeitung mit Wasser erhält man aus 28 direkt das Carbinol 29b, aus dem beim Erhitzen mit Essigsäure die $N$-Schutzgruppe unter Bildung von 5-(Hydroxyphenylmethyl)pyrazol (30a) abgespalten wird. Bei der Oxidation von 29b mit Natriumdichromat in Essigsäure entsteht wiederum unter gleichzeitiger Abspaltung der $\mathrm{N}$ Schutzgruppe 5-Benzoylpyrazol (30 b).

Die angeführten Beispiele belegen, daß bei der Anwendung TMS-substituierter Azole in der Synthese die Dimethylsulfamoyl-Schutzgruppe wegen ihrer Stabilität gegenüber Metallierungsagenzien und wegen ihrer unter milden Bedingungen erfolgenden Abspaltung sehr vorteilhaft ist.

Der Deutschen Forschungsgemeinschaft und dem Fonds der Chemischen Industrie danken wir für die Unterstützung dieser Arbeit; Herrn Dr. D. Krimmer danken wir für geschickte experimentelle Mithilfe

\section{Experimenteller Teil}

${ }^{1}$ H-NMR: Varian T 60 und EM 360, Bruker WP 80, HX 90 und CXP 300, TMS als interner Standard. - IR: Perkin-Elmer-Registrierphotometer 457. - Präparative Säulenchromatographie: Glassäulen verschiedener Dimensionen mit Kieselgel 60, 230-400 mesh ASTM (Fa. Macherey-Nagel). - Gaschromatographie für Umsatzbestimmungen: Hewlett-Packard, 5700 A mit FID, Spectra-
Physics Minigrator. Trägergas: $30 \mathrm{ml} /$ min Stickstoff, Glassäulen 2.3 $\mathrm{m} \times 2 \mathrm{~mm}$, Phasen mit jeweils 5\% OV 17, OV 101 bzw. CW 20 $\mathrm{M}$ auf Chromosorb W, 100-120 mesh.

Allgemeines: Alle Reaktionen wurden unter Stickstoff und unter Feuchtigkeitsausschluß durchgeführt. - Als charakteristische spektroskopische Daten werden die ${ }^{1} \mathrm{H}-\mathrm{NMR}-$ Protonensignale der Imidazole in 2-, 4- und 5-Stellung angegeben sowie die IR-Schwingungsfrequenzen der Carbonylgruppen der Substituenten. Die $\mathrm{NCH}_{3}$-Protonen erscheinen jeweils bei $\delta=3.46-4.34(\mathrm{~s}, 3 \mathrm{H})$ und die $\mathrm{Si}\left(\mathrm{CH}_{3}\right)_{3}$-Protonen bei $\delta=0.31-0.40(\mathrm{~s}, 9 \mathrm{H})$.

1-Methyl-2,5-bis(trimethylsilyl)imidazol (3a): Man tropft $\mathrm{zu}$ $12.8 \mathrm{~g}$ (0.156 mol) 1-Methylimidazol (1 a), $36.3 \mathrm{~g}$ (0.312 mol) 1,2Bis(dimethylamino)ethan (TMEDA) und $100 \mathrm{ml}$ absol. Diethylether eine Lösung von $195 \mathrm{ml}(0.312 \mathrm{~mol}) 1.6 \mathrm{~N}$ Butyllithium in Hexan - zunächst $19.5 \mathrm{ml}$ bei $-30^{\circ} \mathrm{C}$, dann $175.5 \mathrm{ml}$ bei $-40^{\circ} \mathrm{C}$ - und rührt $2 \mathrm{~h}$ bei $-40^{\circ} \mathrm{C}$. Nach Erwärmen auf Raumtemp. innerhalb von $2 \mathrm{~h}$ tropft man zu dem Gemisch eine Lösung von $33.8 \mathrm{~g}(0.312 \mathrm{~mol})$ Trimethylsilylchlorid (TMSC) in $100 \mathrm{ml}$ absol. Ether, rührt 2 h unter Rückfluß, läßt ca. 12 h bei Raumtemp. stehen, filtriert und engt das Filtrat im Rotationsverdampfer i.Vak. ein. Aus dem festen Rückstand werden die bei 0.01 Torr flüchtigen Anteile langsam abdestilliert und verworfen, dann wird das Produkt unter Erwärmen bis zu $110^{\circ} \mathrm{C}$ (Badtemp.) in eine 1-1-Blase sublimiert und das Sublimat aus $110 \mathrm{ml}$ absol. Hexan umkristallisiert, Ausb. $24.0 \mathrm{~g}(68 \%)$, Schmp. $127-133^{\circ} \mathrm{C}$ [ Lit. $^{15 a)} 119-121^{\circ} \mathrm{C}$ (Pen$\tan )]$.

\section{1-Methyl-5-(trimethylsilyl)imidazol (4a)}

a) Man läßt $17.5 \mathrm{~g}(0.077 \mathrm{~mol}) \mathbf{3 a}$ in $100 \mathrm{ml}$ Methanol $1 \mathrm{~h}$ bei Raumtemp. stehen und kristallisiert das nach Abdestillieren des Methanols bei $35^{\circ} \mathrm{C}$ zurückbleibende Produkt aus Pentan um, Ausb. $10.1 \mathrm{~g} \mathrm{(85 \% ),} \mathrm{Schmp.} 54^{\circ} \mathrm{C}$ (Lit. ${ }^{15 a)} \mathrm{Sdp} .58^{\circ} \mathrm{C} / 10$ Torr). ${ }^{1} \mathrm{H}-\mathrm{NMR}\left(\mathrm{CDCl}_{3}\right): \delta=7.17(\mathrm{~s}, 1 \mathrm{H}, 4-\mathrm{H}), 7.58(\mathrm{~s}, 1 \mathrm{H}, 2-\mathrm{H})$.

b) Man tropft unter Rühren innerhalb $2 \mathrm{~h}$ zu $12.15 \mathrm{~g}(0.50 \mathrm{~mol})$ Magnesiumgrieß (zuvor $2 \mathrm{~h}$ bei $50^{\circ} \mathrm{C}$ mit lod geätzt) und $54.3 \mathrm{~g}$ $(0.50 \mathrm{~mol}) \mathrm{TMSC}$ in $200 \mathrm{ml}$ Tetrahydrofuran die Lösung von $46.62 \mathrm{~g} \mathrm{(0.40} \mathrm{mol)} \mathrm{5-Chlor-1-methylimidazol} \mathrm{(5)} \mathrm{in} 100 \mathrm{ml}$ Tetrahydrofuran, rührt $3 \mathrm{~h}$ unter Rückfluß, gieBt das Gemisch auf $500 \mathrm{ml}$ $0.5 \mathrm{~N}$ Natriumhydrogencarbonat-Lösung, extrahiert zweimal mit jeweils $300 \mathrm{ml}$ Ether, trocknet die Extrakte mit Magnesiumsulfat, engt im Rotationsverdampfer ein, destilliert den öligen Rückstand bei Sdp. $56^{\circ} \mathrm{C} / 0.02$ Torr und kristallisiert das Destillat aus Pentan um, Ausb. $49.82 \mathrm{~g}(81 \%)$, Schmp. $52-53^{\circ} \mathrm{C}$

2,4,5-Tribrom-1-methylimidazol (6): Man tropft zu $34.25 \mathrm{~g}(0.42$ mol) $1 \mathrm{a}$ und $400.0 \mathrm{~g}(4.38 \mathrm{~mol})$ Natriumacetat in 31 Eisessig innerhalb $40 \mathrm{~min} 1 / 3$ der Lösung von $192.0 \mathrm{~g}$ (1.25 mol) Brom in $400 \mathrm{ml}$ Eisessig, gibt $100.0 \mathrm{~g}(1.22 \mathrm{~mol})$ Natriumacetat zu, tropft innerhalb $40 \mathrm{~min}$ die restliche Bromlösung zu und rührt $2.5 \mathrm{~h}$ bei Raumtemp. Dann wird der Eisessig im Rotationsverdampfer bei $50^{\circ} \mathrm{C}$ entfernt, der Rückstand mit 3.51 Wasser aufgeschlämmt, der verbleibende Niederschlag mit 31 Wasser neutral gewaschen und im Exsikkator über Phosphorpentoxid und Kaliumhydroxid bei 15 Torr getrocknet. Zur vollständigen Entfernung des Wassers wird das Produkt in Dichlormethan gelöst, noch vorhandenes Wasser abgetrennt, die organische Phase mit Natriumsulfat getrocknet, im Rotationsverdampfer eingeengt und der Rückstand ( $49.3 \mathrm{~g}$ farblose Kristalle) aus $360 \mathrm{ml}$ Ethylacetat/Hexan (1:5) umkristallisiert, Ausb. $37.5 \mathrm{~g}$, nach Aufarbeiten der Mutterlauge insgesamt $43.9 \mathrm{~g}$ (33\%), Schmp. $93-95^{\circ} \mathrm{C}$ [ Lit. $^{17 \text { a) }} 93-94.5^{\circ} \mathrm{C}$ (Ether)].

4,5-Dibrom-1-methylimidazol (7): Unter leichtem Erwärmen tropft man zu der Lösung von $1.055 \mathrm{~g}(3.31 \mathrm{mmol}) 6$ in $2 \mathrm{ml}$ Ethanol die Lösung von $0.872 \mathrm{~g}(3.33 \mathrm{mmol})$ Triphenylphosphan in $3.4 \mathrm{ml}$ 
Ethanol, erhitzt 45 min unter Rückfluß, engt im Rotationsverdampfer ein, gibt zum Rückstand $10 \mathrm{ml} 0.5 \mathrm{~N} \mathrm{NaOH}$ und rühtt $1 \mathrm{~h}$ bei Raumtemp. Nach dreimaliger Extraktion mit jeweils $50 \mathrm{ml}$ Ether werden die vereinigten Etherextrakte mit $20 \mathrm{ml}$ Wasser gewaschen, mit Magnesiumsulfat getrocknet und im Rotationsverdampfer eingeengt. Der Rückstand ( $1.613 \mathrm{~g}$ farblose ölige Kristalle) wird zweimal bei $60^{\circ} \mathrm{C} / 0.01$ Torr sublimiert, Ausb. $0.636 \mathrm{~g} \mathrm{(80 \% ),} \mathrm{Schmp.}$ $81.5-82^{\circ} \mathrm{C}$ [Lit. ${ }^{18)} 80-81^{\circ} \mathrm{C}$ (Benzol/leichtes Petroleum)].

4-Brom-1-methyl-5-(trimethylsilyl)imidazol (8): Nach Lit. ${ }^{19 a)}$ wird zu $1.52 \mathrm{~g}$ (62.5 mmol) Magnesium-Spänen und $6.79 \mathrm{~g}$ (62.5 mmol) TMSC in $30 \mathrm{ml}$ Hexamethylphosphorsäuretriamid (HMPT) unter Rühren bei $80^{\circ} \mathrm{C}$ innerhalb $2 \mathrm{~h}$ die Lösung von $6.0 \mathrm{~g}(25.0 \mathrm{mmol})$ 7 in $15 \mathrm{ml} \mathrm{HMPT}$ getropft, anschließend $24 \mathrm{~h}$ bei $80^{\circ} \mathrm{C}$ gerührt und aufgearbeitet; Ausb. 4.5 g (77\%), Sdp. $83^{\circ} \mathrm{C} / 0.002$ Torr. $-{ }^{1} \mathrm{H}-\mathrm{NMR}$ $\left(\mathrm{CDCl}_{3}\right.$, TMS extern): $\delta=7.46(\mathrm{~s}, 1 \mathrm{H}, 2-\mathrm{H})$.

$$
\begin{aligned}
& \mathrm{C}_{7} \mathrm{H}_{13} \mathrm{BrN}_{2} \mathrm{Si}(233.2) \\
& \text { Ber. C } 36.05 \mathrm{H} 5.62 \mathrm{Br} 34.27 \mathrm{~N} 12.01 \\
& \text { Gef. C } 36.44 \mathrm{H} 5.82 \mathrm{Br} 33.18 \mathrm{~N} 12.11
\end{aligned}
$$

1-Methyl-4,5-bis(trimethylsilyl)imidazol (9): $\mathrm{Zu} 17.01 \mathrm{~g}(0.70 \mathrm{~mol})$ Magnesiumgrieß (zuvor $2 \mathrm{~h}$ bei $50^{\circ} \mathrm{C}$ mit Iod geätzt), $91.26 \mathrm{~g}(0.84$ mol) TMSC und $450 \mathrm{ml}$ Tetrahydrofuran gibt man unter Argon $1.5 \mathrm{~g}(0.047 \mathrm{~mol})$ kristallines 6 und tropft innerhalb $5 \mathrm{~h}$ unter Kühlen auf $2-5^{\circ} \mathrm{C}$ die Lösung von $43.6 \mathrm{~g}(0.137 \mathrm{~mol}) 6$ in $225 \mathrm{ml}$ Tetrahydrofuran zu. Es wird ca. $12 \mathrm{~h}$ gerührt, das Gemisch dann in $550 \mathrm{ml}$ einer ges. Natriumhydrogencarbonat-Lösung und $250 \mathrm{~g}$ Eis eingerührt und vom nicht umgesetzten Magnesium abfiltriert. Die organische Phase wird abgetrennt und die wäßrige Phase fünfmal mit jeweils $300 \mathrm{ml}$ Ether extrahiert. Die vereinigten organischen Phasen werden auf $500 \mathrm{ml}$ eingeengt, mit Natriumsulfat getrocknet und die Lösungsmittel im Rotationsverdampfer bei $70^{\circ} \mathrm{C}$ abdestilliert; der Rückstand wird über eine 20-cm-Vigreux-Kolonne i. Vak. destilliert, Ausb. 17.0 g, Sdp. $58^{\circ} \mathrm{C} / 0.001$ Torr, Schmp. $<49^{\circ} \mathrm{C}$. Der Vorlauf $(7.7 \mathrm{~g})$ wird über eine Spaltrohrkolonne destilliert, Ausb. 2.9 g, Sdp. $150^{\circ} \mathrm{C} / 15$ Torr, Gesamtausb. $19.9 \mathrm{~g} \mathrm{(63 \% ),} \mathrm{Schmp.}$ $52.5-53.3^{\circ} \mathrm{C}$ (Pentan). $-{ }^{1} \mathrm{H}-\mathrm{NMR}\left(\mathrm{CDCl}_{3}\right): \delta=7.65(\mathrm{~s}, 1 \mathrm{H}, 2-\mathrm{H})$.

$$
\begin{array}{ll}
\mathrm{C}_{10} \mathrm{H}_{22} \mathrm{~N}_{2} \mathrm{Si}_{2} \text { (226.5) } & \text { Ber. C 53.04 H } 9.79 \mathrm{~N} 12.37 \\
& \text { Gef. C 52.95 H } 9.73 \mathrm{~N} 12.13
\end{array}
$$

1-Methyl-4-(trimethylsilyl)imidazol (11 a): Wie bei der Darstellung von $4 \mathrm{a}$ nach $\mathrm{b})$ beschrieben aus $5.0 \mathrm{~g}(0.208 \mathrm{~mol}) \mathrm{mit}$ Iod vorbehandeltem Magnesium, $85 \mathrm{ml}$ Tetrahydrofuran, $26.5 \mathrm{ml}$ $(0.208 \mathrm{~mol})$ TMSC und $19.54 \mathrm{~g}(0.167 \mathrm{~mol})$ 4-Chlor-1-methylimidazol (10) in $45 \mathrm{ml}$ Tetrahydrofuran. Es wird $25 \mathrm{~h}$ unter Rückfluß erhitzt und gerührt. Nach Aufarbeiten mit $500 \mathrm{ml} 0.5 \mathrm{~N}$ Natriumhydrogencarbonat-Lösung und zweimaliger Extraktion mit jeweils $300 \mathrm{ml}$ Ether werden die Extrakte mit Magnesiumsulfat getrocknet und im Rotationsverdampfer eingeengt, der ölige Rückstand wird i.Vak. destilliert, Ausb. $15.02 \mathrm{~g}(58 \%)$, Sdp. $56^{\circ} \mathrm{C} / 0.02$ Torr. $-{ }^{1} \mathrm{H}$ NMR $\left(\mathrm{CDCl}_{3}\right): \delta=7.03(\mathrm{~s}, 1 \mathrm{H}, 5-\mathrm{H}), 7.60(\mathrm{~s}, 1 \mathrm{H}, 2-\mathrm{H})$.

$$
\begin{array}{lll}
\mathrm{C}_{7} \mathrm{H}_{14} \mathrm{~N}_{2} \mathrm{Si} \text { (154.3) } & \text { Ber. C } 54.49 \text { H } 9.15 \text { N } 18.14 \\
& \text { Gef. C } 54.61 \text { H } 9.40 \text { N } 18.30
\end{array}
$$

Unkatalysierte Umsetzungen von $\mathbf{3 a}$ mit Elektrophilen (zu Tab. 1 und Schema 2): In einem ausgeheizten Zweihalskolben wird 3a in Substanz oder gelöst in Tetrachlormethan - bei Raumtemp. mit dem Elektrophil versetzt und bei der angegebenen Temp. gerührt. Nach Entfernen des Lösungsmittels im Rotationsverdampfer (wenn erforderlich) wird das Produkt i.Vak. destilliert (Tab. 6).

Caesiumfluorid-katalysierte Umsetzungen von 5-(Trimethylsilyl)substituierten 1-Methylimidazolen 4a, 12 und 14 mit Benzaldehyd (A). Benzoylfluorid (B) bzw. Fluorameisensäure-isobutylester (C) (zu Tab. 2 und Schema 2): In einem ausgeheizten Rundkolben mit Quickfit-Verschluß wird im Stickstoff-Kasten CsF eingewogen, das
Tab. 6. Unkatalysierte Umsetzung von 3a mit Elcktrophilen ${ }^{\text {a) }}$

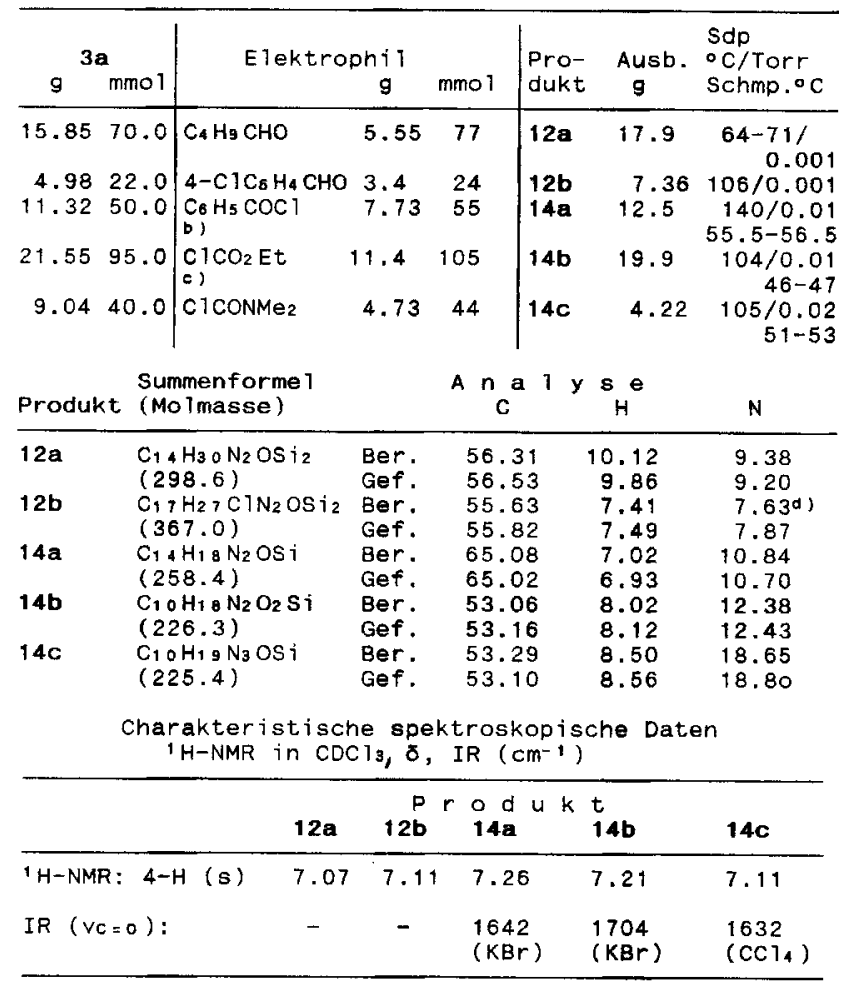

a) Reaktionsbedingungen und Ausbeuten (\%) siehe Tab. 1. - ${ }^{b, c)}$ Sowie $\mathrm{CCl}_{4}$ : b) $60 \mathrm{ml}$, ${ }^{\text {c }} 75 \mathrm{ml}$. - d) $\mathrm{Cl}$ : Ber. 9.66, Gef. 9.66.

Elektrophil in Substanz, eine Lösung von 4a, 12 bzw. 14 in Dimethylformamid zugegeben und bei der angegebenen Temp. bis zur vollständigen Umsetzung (GC- oder dünnschichtchromatographisch bestimmt) getührt. - Aufarbeitung: a) Umsetzungen mit $A$ : Bei der angegebenen Reaktionstemp. wird das Reaktionsgemisch mit 1 Äquivalent Wasser pro TMS-Gruppe versetzt, 5 min gerührt, auf Wasser gegossen, mit Ethylacetat oder Chloroform extrahiert, der Extrakt mit Wasser gewaschen, mit Magnesiumsulfat getrocknet und im Rotationsverdampfer eingeengt. Die ölig anfallenden Alkohole 13 bzw. 15 werden durch Anreiben mit Ether zur Kristallisation gebracht und umkristallisiert; b) Umsetzung von 14b mit B: Das Reaktionsgemisch wird nicht mit Wasser, sondern mit $120 \mathrm{ml}$ Ether versetzt, filtriert, das Filtrat eingeengt, der Rückstand mit Ethylacetat aufgenommen und über eine $10 \mathrm{~cm}$ lange Kieselgelsäule filtriert; c) Umsetzung von $14 \mathbf{b}$ mit $C$ : Das Rohprodukt wird bei $100-150^{\circ} \mathrm{C} / 0.01$ Torr über ein Kugelrohr destilliert und dann über Kieselgel mit Ethylacetat/Petrolether (1:3) chromatographiert (Tab. 7).

Umsetzungen der 5-(Trimethylsilyl)-substituierten 1-Methylimidazole 4a, 12a und 14a,b (Tab. 3, Schema 2) sowie der 4-(Trimethylsilyl)-substituierten 1-Methylimidazole 11 a und $20 \mathrm{a}$ mit Benzoylchlorid (D) bzw. Chlorameisensäure-ethylester (E) (Tab. 4, Schema 3)

Umsetzungen von 4a und 11 a mit $D$ oder $E$ in Gegenwart von Triethylamin in Acetonitril: In einem ausgeheizten Reaktionskolben wird unter Eiskühlung und Rühren zu der Lösung des TMS-substituierten 1-Methylimidazols in Acetonitril zuerst D oder E, dann absol. Triethylamin getropft, anschließend wird unter Feuchtigkeitsausschluß bei der angegebenen Temp. gerührt und wie folgt aufgearbeitet: a) Umsetzungen mit D: Das Reaktionsgemisch wird 
Tab. 7. CsF-katalysierte Umsetzungen von $4 \mathrm{a}, 12$ und 14 mit Benzaldehyd (A), Benzoylfluorid (B) bzw. Fluorameisensäure-isobutylester $(C)^{\text {a) }}$

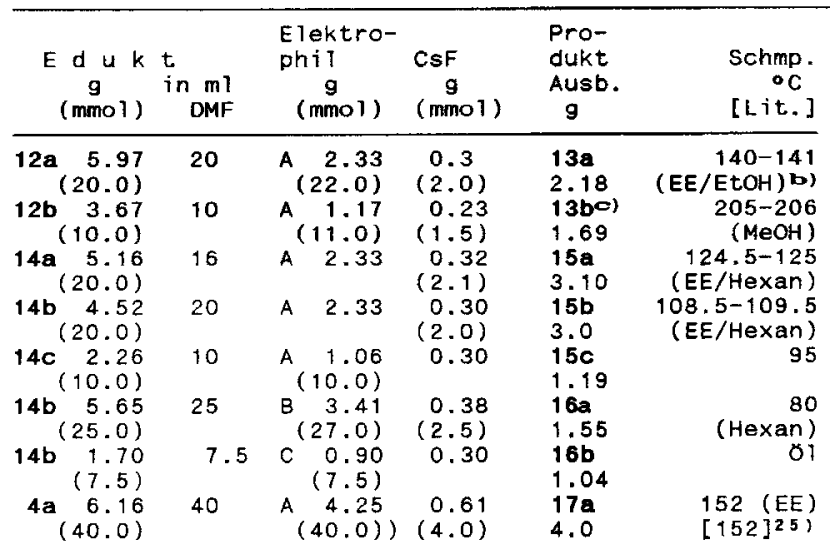

\begin{tabular}{|c|c|c|c|c|c|}
\hline Produkt & $\begin{array}{l}\text { Summenforme } 1 \\
\text { (Molmasse) }\end{array}$ & & $\mathrm{A}_{\mathrm{C}} \mathrm{a}$ & ${ }_{H}^{y} s e$ & $\mathrm{~N}$ \\
\hline $13 a$ & $\begin{array}{l}\mathrm{C}_{15} \mathrm{H}_{2} \mathrm{ON}_{2} \mathrm{O}_{2} \\
(260,3)\end{array}$ & $\begin{array}{l}\text { Ber. } \\
\text { Gef. }\end{array}$ & $\begin{array}{l}69.20 \\
69.28\end{array}$ & $\begin{array}{l}7.75 \\
7.91\end{array}$ & $\begin{array}{l}10.76 \\
10.55\end{array}$ \\
\hline $13 b$ & $\begin{array}{l}\mathrm{C}_{18 \mathrm{H}_{1}} \mathrm{Cl}_{1 \mathrm{~N}_{2} \mathrm{O}_{2}} \\
(328.8)\end{array}$ & $\begin{array}{l}\text { Ber: } \\
\text { Gef: }\end{array}$ & $\begin{array}{l}65.74 \\
65.60\end{array}$ & $\begin{array}{l}5.21 \\
5.26\end{array}$ & $\begin{array}{l}8.52 \mathrm{~d}) \\
8.26\end{array}$ \\
\hline $15 a$ & $\begin{array}{l}\mathrm{C}_{1} 8 \mathrm{H}_{1} 6 \mathrm{~N}_{2} \mathrm{O}_{2} \\
(292.3)\end{array}$ & $\begin{array}{l}\text { Ber. } \\
\text { Gef. }\end{array}$ & $\begin{array}{l}73.95 \\
73.77\end{array}$ & $\begin{array}{l}5.52 \\
5.57\end{array}$ & $\begin{array}{l}9.58 \\
9.30\end{array}$ \\
\hline $15 b$ & $\begin{array}{l}\mathrm{C}_{1} 4 \mathrm{H}_{1} \mathrm{H}_{2} \mathrm{O}_{3} \\
(260.3)\end{array}$ & $\begin{array}{l}\text { Ber. } \\
\text { Gef. }\end{array}$ & $\begin{array}{l}64.60 \\
64.48\end{array}$ & $\begin{array}{l}6.20 \\
6.19\end{array}$ & $\begin{array}{l}10.76 \\
10.86\end{array}$ \\
\hline $15 c$ & $\begin{array}{l}\mathrm{C}_{1}+\mathrm{H}_{1} 7 \mathrm{~N}_{3} \mathrm{O}_{2} \\
(259.3)\end{array}$ & $\begin{array}{l}\text { Ber. } \\
\text { Gef. }\end{array}$ & $\begin{array}{l}64.85 \\
64.60\end{array}$ & $\begin{array}{l}6.61 \\
6.70\end{array}$ & $\begin{array}{l}16.20 \\
15.99\end{array}$ \\
\hline $16 a$ & $\begin{array}{l}\mathrm{C}_{1}{ }_{4} \mathrm{H}_{1}{ }_{4} \mathrm{~N}_{2} \mathrm{O}_{3} \\
(258.3)\end{array}$ & $\begin{array}{l}\text { Ber. } \\
\text { Gef: }\end{array}$ & $\begin{array}{l}65.11 \\
65.29\end{array}$ & $\begin{array}{l}5.46 \\
5.35\end{array}$ & $\begin{array}{l}10.85 \\
10.85\end{array}$ \\
\hline $16 b$ & $\begin{array}{l}\mathrm{C}_{12} \mathrm{H}_{1} 8_{8} \mathrm{~N}_{2} \mathrm{O}_{4} \\
(254.3)\end{array}$ & $\begin{array}{l}\text { Ber. } \\
\text { Gef. }\end{array}$ & $\begin{array}{l}56.68 \\
56.76\end{array}$ & $\begin{array}{l}7.13 \\
7.04\end{array}$ & $\begin{array}{l}11.02 \\
11.31\end{array}$ \\
\hline $17 \mathrm{a}$ & $\begin{array}{l}\mathrm{C}_{1}{ }_{1} \mathrm{H}_{1} \mathrm{~N}_{2} \mathrm{O} \\
(188.2)\end{array}$ & $\begin{array}{l}\text { Ber. } \\
\text { Gef. }\end{array}$ & $\begin{array}{l}70.19 \\
70.65\end{array}$ & $\begin{array}{l}6.42 \\
6.47\end{array}$ & $\begin{array}{l}14.88 \\
14.81\end{array}$ \\
\hline
\end{tabular}

Charakteristische spektroskopische Daten $1 \mathrm{H}$-NMR in $\mathrm{CDCl}_{3} \mathrm{\delta}$, IR $\left(\mathrm{cm}^{-1}\right)$

\begin{tabular}{|c|c|c|c|c|c|}
\hline $\begin{array}{l}\text { Pro- } \\
\text { dukt }\end{array}$ & $\begin{array}{l}1 H-N M R \\
4-H \text { (s) }\end{array}$ & $\begin{array}{l}\text { IR } \\
\left(\begin{array}{l}\text { V }=0) \\
\text { Keton }\end{array}\right.\end{array}$ & $\begin{array}{l}\text { Pro- } \\
\text { dukt }\end{array}$ & $\begin{array}{l}1 \mathrm{H}-\mathrm{NMR} \\
4-H \text { (s) }\end{array}$ & $\begin{array}{l}\text { IR } \\
(V c=0) \\
\text { Keton }\end{array}$ \\
\hline $\begin{array}{l}13 a \\
13 b \\
15 a \\
15 b \\
15 c\end{array}$ & $\begin{array}{l}6.440) \\
6.400) \\
6.77 \\
6.74 \\
6.66\end{array}$ & $\begin{array}{l}- \\
- \\
1638 \\
(\mathrm{KBr}) \\
1704 \\
(\mathrm{KBr}) \\
1629 \\
(\mathrm{KBr})\end{array}$ & $\begin{array}{l}16 a \\
16 b \\
\left.17 a^{e}\right)\end{array}$ & $\begin{array}{l}7.5-7.75 \\
(\mathrm{~m}, 4-\mathrm{H}, \\
\left.5-\mathrm{CH}_{6} \mathrm{Hs}_{\mathrm{s}}\right) \\
7.81 \\
6.57+ \\
7.58 \\
(\mathrm{~s}, 2-\mathrm{H})\end{array}$ & $\begin{array}{l}1638+ \\
1716 \text { (Ester) } \\
\text { (KBr) } \\
1714 \\
\text { (f1.kap.) } \\
=\end{array}$ \\
\hline
\end{tabular}

a) Reaktionsbedingungen und Ausbeuten (\%) siehe Tab. 2. ${ }^{\text {b) }} \mathrm{EE}=$ Essigsäure-ethylester. - ${ }^{\mathrm{c})}$ Nach Eingießen des Reaktionsgemisches in Wasser wurde der nach Zugeben von $15 \mathrm{ml}$ Ethylacetat ausgefallene Niederschlag abgesaugt, mit Wasser und Ether gewaschen, getrocknet und mit Methanol umkristallisiert. $-{ }^{\text {d) }} \mathrm{Cl}$ : Ber. 10.80, Gef. 10.85. - ${ }^{\text {e) }}{ }^{1} \mathrm{H}-\mathrm{NMR}$ in $\mathrm{CD}_{3} \mathrm{OD}$.

auf absol. Ether gegossen, filtriert und der abfiltrierte Niederschlag mit Ether gewaschen; b) Umsetzungen mit E: Das Reaktionsgemisch wird auf Wasser gegossen und zweimal ausgeethert, die vereinigten Extrakte werden mit Wasser gewaschen und mit Magnesiumsulfat getrocknet. Die nach a) und b) erhaltenen etherischen Lösungen werden jeweils im Rotationsverdampfer eingeengt, der Rückstand wird i.Hochvak. destilliert oder umkristallisiert.

Umsetzungen von 11, 12a, 14a,b und 20 a mit D: Das TMS-substituierte 1-Methylimidazol und D werden in einem Rundkolben mit aufgesetzter kleiner Spiegelkolonne unter Rühren bis zum vollständigen Umsatz (anhand des abdestillierenden TMSC bestimmt) erhitzt, dann wird überschüssiges $\mathrm{D}$ bei 0.01 Torr (Badtemp. bis $100^{\circ} \mathrm{C}$ ) abdestilliert, der Rückstand in Ethylacetat, Aceton oder
Methanol aufgenommen und über Kieselgel chromatographiert und/oder umkristallisiert (Tab. 8).

Tab. 8. Umsetzungen der 5-(Trimethylsilyl)-substituierten 1-Methylimidazole 4, 12a und 14a,b sowie der 4-(Trimethylsilyl)-substituierten 1-Methylimidazole 11a und 20a mit Benzoylchlorid (D) bzw. Chlorameisensäure-ethylester $(E)^{\text {a) }}$

\begin{tabular}{|c|c|c|c|c|c|}
\hline $\begin{array}{l}\text { Edukt } \\
\qquad \begin{array}{l}\mathbf{g} \\
\text { (mmol) }\end{array}\end{array}$ & $\mid$\begin{tabular}{c} 
Elektro- \\
phi 1 \\
\multicolumn{2}{c}{ g } \\
\\
(mmo 1$)$
\end{tabular} & $\begin{array}{r}\mathrm{MeCN} \\
\mathrm{m}\end{array}$ & $\begin{array}{c}\mathrm{Et}_{3} \mathrm{~N} \\
\mathrm{~g} \\
(\mathrm{mmol})\end{array}$ & $\begin{array}{l}\text { Produkt } \\
\text { Ausb. (g) }\end{array}$ & $\begin{array}{l}\text { Sdp } \\
\text { oc/Torr } \\
\text { schmp. } \\
\text { oc }\end{array}$ \\
\hline 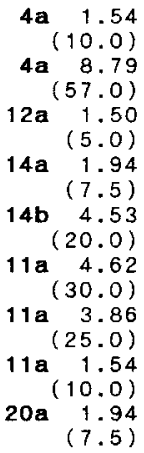 & $\begin{array}{lr}D & 1.55 \\
& (11.0) \\
& 12.31 \\
& (114.0) \\
D & 1.55 \\
& (11.0) \\
D & 1.16 \\
& (8.3) \\
E & 3.09 \\
& (22.0) \\
D & 4.65 \\
& (33.0) \\
E & 5.4 \\
& (50.0) \\
D & 1.55 \\
& (11.0) \\
D & 1.16 \\
& (8.3)\end{array}$ & - & $\begin{array}{c}1.01 \\
(10.0) \\
11.51 \\
(114.0) \\
- \\
-\end{array}$ & $\begin{array}{l}14 a \\
(2.17) \\
14 b b) \\
(7.27) \\
19 \\
(1.37) \\
18 \\
(1.12) \\
16 a \\
(3.05) \\
20 a \\
(5.4) \\
20 b \\
(1.08) \\
20 a \\
(0.84) 8) \\
21 \\
(1.38)\end{array}$ & $\begin{array}{l}56.5-57.5 \\
(\text { Pentan) } \\
78-80 / 0.008 \\
\text { hochviskoses } \\
98-99 \text { Ölo) } \\
(\mathrm{MeOH}) \mathrm{d}) \\
80-810, \mathrm{f}) \\
123-125 / 0.02 \\
68-70(\text { Pentan } \\
70-72 / 0.002 \\
53-54 \text {, Olc) } \\
\text { hochviskoses } \\
147-148 \\
\text { (MeOH/EE) }\end{array}$ \\
\hline
\end{tabular}

\begin{tabular}{|c|c|c|c|c|c|}
\hline $\begin{array}{l}\text { Pro- } \\
\text { dukth) }\end{array}$ & $\begin{array}{l}\text { Summenforme } 1 \\
\text { (Molmasse) }\end{array}$ & & $\mathrm{A}_{\mathrm{C}}^{\mathrm{n}} \mathrm{a}$ & $\mathrm{y}_{\mathrm{H}} \mathrm{e}$ & $\mathrm{N}$ \\
\hline 19 & $\begin{array}{l}\mathrm{C}_{22} \mathrm{H}_{2} 2 \mathrm{~N}_{2} \mathrm{O}_{3} \\
(362.4)\end{array}$ & $\begin{array}{l}\text { Ber. } \\
\text { Gef. }\end{array}$ & $\begin{array}{l}72.91 \\
72.84\end{array}$ & $\begin{array}{l}6.12 \\
6.06\end{array}$ & $\begin{array}{l}7.73 \\
7.69\end{array}$ \\
\hline 18 & $\begin{array}{l}\mathrm{C}_{1} \mathrm{BH}_{1}+\mathrm{N}_{2} \mathrm{O}_{2} \\
(290.3)\end{array}$ & $\begin{array}{l}\text { Ber. } \\
\text { Gef. }\end{array}$ & $\begin{array}{l}74.47 \\
74.42\end{array}$ & $\begin{array}{l}4.86 \\
4.87\end{array}$ & $\begin{array}{l}9.65 \\
9.64\end{array}$ \\
\hline $20 a$ & $\begin{array}{l}\mathrm{C}_{1}+\mathrm{H}_{1} \text { \& } \mathrm{N}_{2} \text { OS } \mathrm{i} \\
(258.4)\end{array}$ & $\begin{array}{l}\text { Ber. } \\
\text { Gef. }\end{array}$ & $\begin{array}{l}65.08 \\
64.84\end{array}$ & $\begin{array}{l}7.02 \\
6.98\end{array}$ & $\begin{array}{l}10.84 \\
10.76\end{array}$ \\
\hline $20 b$ & $\begin{array}{l}\mathrm{C}_{1} \text { a } \mathrm{H}_{1} \text { \& } \mathrm{N}_{2} \mathrm{O}_{2} \mathrm{Si} \\
(226,3)\end{array}$ & $\begin{array}{l}\text { Ber. } \\
\text { Gef. }\end{array}$ & $\begin{array}{l}53.06 \\
52.79\end{array}$ & $\begin{array}{l}8.02 \\
7.75\end{array}$ & $\begin{array}{l}12.38 \\
12.60\end{array}$ \\
\hline 21 & $\begin{array}{l}\mathrm{C}_{1} 8 \mathrm{H}_{1} \mathrm{~N}_{2} \mathrm{O}_{2} \\
(290.3)\end{array}$ & $\begin{array}{l}\text { Ber. } \\
\text { Gef. }\end{array}$ & $\begin{array}{l}74.47 \\
74.26\end{array}$ & $\begin{array}{l}4.86 \\
5.01\end{array}$ & $\begin{array}{l}9.65 \\
9.63\end{array}$ \\
\hline
\end{tabular}

\begin{tabular}{|c|c|c|c|c|c|}
\hline $\begin{array}{l}\text { Pro- } \\
\text { dukt }\end{array}$ & ${ }^{1} \mathrm{H}-\mathrm{NMR}$ & $\begin{array}{l}\text { IR } \\
(v c=0) \\
\text { Keton }\end{array}$ & $\begin{array}{l}\text { Pro- } \\
\text { dukt }\end{array}$ & $\begin{array}{l}1 \mathrm{H}-\mathrm{NMR} \\
5-\mathrm{H} \\
(\mathrm{s})\end{array}$ & $\begin{array}{l}\text { IR } \\
(v c=0) \\
\text { Keton }\end{array}$ \\
\hline 19 & $\begin{array}{l}7.3-7.7 \\
(\mathrm{~m}, 4-\mathrm{H}, \\
2-\mathrm{CHOCOC}_{6} \mathrm{H}_{5} \\
5-\mathrm{COPh})\end{array}$ & $\begin{array}{l}1639+ \\
1716(\text { Ester }) \\
(\mathrm{CC} 14)\end{array}$ & $\begin{array}{l}20 a \\
20 b\end{array}$ & $\begin{array}{l}7.18 \\
7.13\end{array}$ & $\begin{array}{l}1644 \\
(\mathrm{KBr}) \\
1707 \\
(\mathrm{fl} \text { l.kap. })\end{array}$ \\
\hline 18 & $\begin{array}{l}7.46-7.77 \\
(m, 4-H, \\
2,5-\mathrm{COPh})\end{array}$ & $\begin{array}{l}1641+ \\
1660 \\
(\mathrm{KBr})\end{array}$ & 21 & 6.67 & $\begin{array}{l}1637 \\
(\mathrm{KBr})\end{array}$ \\
\hline
\end{tabular}

a) Reaktionsbedingungen und Ausbeuten (\%) siehe Tab. 3 und 4.

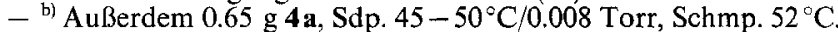
- ${ }^{c)}$ Nach Chromatographie an $\mathrm{SiO}_{2}$ mit Ethylacetat/Petrolether (EE/PE) $(1: 2)$ - d) Nach Chromatographie an $\mathrm{SiO}_{2}$ mit EE/PE $(1: 6)$. - e) Bildet eine metastabile Kristallmodifikation mit Schmp. $73^{\circ} \mathrm{C}$ (Hexan). - ${ }^{1}{ }^{1} \mathrm{H}$-NMR-spektroskopisch identisch mit dem aus $14 \mathrm{~b}$ und Benzoylfluorid/CsF erhaltenen Produkt. - g) Isoliert nach Chromatographie an $\mathrm{SiO}_{2}$ mit EE/PE (1:4). - ${ }^{\text {h) }}$ 14a,b s. Tab. 6, 16a s. Tab. 7.

Umsetzungen von 4,5-Bis(trimethylsilyl)- (9) und -2-(4-chlorbenzoyl)-1-methylimidazol (23b) mit Benzaldehyd und Aroylhalogeniden (zu Tab, 5 und Schema 3)

1. Umsetzungen von 9: a) Mit Benzaldehyd: $\mathrm{Zu}$ der Suspension von $0.38 \mathrm{~g}(2.5 \mathrm{mmol}) \mathrm{CsF}$ in $2.5 \mathrm{ml}$ Dimethylformamid gibt man $0.587 \mathrm{~g}(5.53 \mathrm{mmol})$ Benzaldehyd und tropft bei $60^{\circ} \mathrm{C}$ innerhalb von $11 \mathrm{~min}$ die Lösung von $1.132 \mathrm{~g}(5.0 \mathrm{mmol}) 9$ in $2.5 \mathrm{ml}$ Dimethylformamid zu. Nach $25 \mathrm{~min}$. Rühren bei $60^{\circ} \mathrm{C}$ wird das Reaktionsgemisch auf $0^{\circ} \mathrm{C}$ abgekühlt, mit $15 \mathrm{ml}$ Ether versetzt und abgesaugt. Aus dem Filtrat werden die leichtflüchtigen Anteile zu- 
nächst bei 15 Torr, dann bei 0.001 Torr und maximal $40^{\circ} \mathrm{C}$ abdestilliert, Ausb. $1.51 \mathrm{~g} \mathrm{(91 \% )} \mathrm{1-Methyl-5-[phenyl(trimethylsil-}$ oxy)methyl]-4-(trimethylsilyl)imidazol (22a) als gelbliches Öl (Reinheit nach GC: 96proz.). - ${ }^{1} \mathrm{H}-\mathrm{NMR}$ (CDCl 3 , TMS ext.): $\delta=$ 7.45 (s, 1 H, 2-H). Nach 18stdg. Rühren von 22a mit $0.46 \mathrm{~g}(10.0$ mmol) Ameisensäure und $10 \mathrm{ml}$ Ethanol bei Raumtemp. werden die leichtflüchtigen Anteile wie voranstehend abgetrennt. Der Rückstand wird in Ether aufgenommen, die Lösung mit $10 \mathrm{ml}$ ges. Natriumhydrogencarbonat-Lösung neutralisiert und mit Ethylacetat, Ether und Dichlormethan extrahiert. Nach Trocknen der vereinigten Extrakte mit Natriumsulfat/Kaliumcarbonat und Entfernen der Lösungsmittel im Rotationsverdampfer Ausb. $1.04 \mathrm{~g}$ 5-(Hydroxyphenylmethyl)-1-methyl-4-(trimethylsilyl)imidazol (22b), Schmp. $168-169^{\circ} \mathrm{C}\left[{ }^{1} \mathrm{H}-\mathrm{NMR}\left(\mathrm{CDCl}_{3}, \mathrm{TMS}\right.\right.$ ext.): $\left.\delta=7.18(\mathrm{~s}, 1 \mathrm{H}, 2-\mathrm{H})\right]$, aus Ether umkristallisiert Ausb. $0.828 \mathrm{~g}(64 \%)$, Schmp. $169-171^{\circ} \mathrm{C}$.

$$
\mathrm{C}_{14} \mathrm{H}_{20} \mathrm{~N}_{2} \mathrm{OSi}(260.4) \quad \text { Ber. C } 64.57 \mathrm{H} 7.74 \mathrm{~N} 10.76
$$$$
\text { Gef. C } 64.70 \text { H } 7.59 \text { N } 10.77
$$

b) Mit 4-Chlorbenzoylchlorid: $\mathrm{Zu} 572.0 \mathrm{mg}$ (2.53 mmol) 9 in $5 \mathrm{ml}$ Acetonitril werden unter Eiskühlung und Rühren innerhalb 6 min $440.8 \mathrm{mg}$ ( $2.52 \mathrm{mmol}$ ) 4-Chlorbenzoylchlorid und dann innerhalb $4 \mathrm{~min} 252.2 \mathrm{mg}$ ( $2.49 \mathrm{mmol}$ ) Triethylamin getropft. Nach Erwärmen auf Raumtemp. innerhalb 10 min wird das rotorange Gemisch $18 \mathrm{~h}$ bei Raumtemp. gerührt, mit $1 \mathrm{ml}$ absol. Methanol versetzt, weitere $3.5 \mathrm{~h}$ bei Raumtemp. gerührt und im Rotationsverdampfer eingeengt. Der Rückstand wird mit Ether versetzt, vom Unlöslichen wird abfiltriert, das Filtrat im Rotationsverdampfer bei $30^{\circ} \mathrm{C}$ zur Trockne eingeengt, der Rückstand ( $881 \mathrm{mg}$ hellbraune Kristalle) aus Pentan umkristallisiert (Ausb. $378 \mathrm{mg}$ ) und die Mutterlauge über Kieselgel mit Petrolether/Ethylacetat (15:1) chromatographiert (Ausb. $262 \mathrm{mg}$ ), Gesamtausb. $640 \mathrm{mg}$ (69\%) 2-(4-Chlorbenzoyl)-1-methyl-4,5-bis(trimethylsilyl)imidazol (23b), Schmp. $121^{\circ} \mathrm{C}$ (Pentan).

$$
\begin{aligned}
& \mathrm{C}_{17} \mathrm{H}_{25} \mathrm{ClN}_{2} \mathrm{OSi}_{2} \text { (365.0) } \\
& \text { Ber. C } 55.94 \mathrm{H} 6.90 \mathrm{Cl} 9.71 \text { N } 7.67 \\
& \text { Gef. C } 56.10 \mathrm{H} 7.08 \mathrm{Cl} 9.68 \mathrm{~N} 7.74
\end{aligned}
$$

c) Mit Benzoylchlorid: $\mathrm{Zu} 4.53 \mathrm{~g}(0.020 \mathrm{~mol}) 9$ und $4.05 \mathrm{~g}(0.040$ mol) Triethylamin in $35 \mathrm{ml}$ Acetonitril werden bei $50^{\circ} \mathrm{C}$ innerhalb $1.5 \mathrm{~h}$ unter Rühren $3.09 \mathrm{~g}(0.022 \mathrm{~mol})$ Benzoylchlorid getropft. Dann wird $17 \mathrm{~h}$ gerührt, bei $50^{\circ} \mathrm{C}$ das Gemisch mit $20 \mathrm{ml}$ Methanol versetzt, weitere $3 \mathrm{~h}$ bei $50^{\circ} \mathrm{C}$ gerührt und im Rotationsverdampfer zunächst bei Normaldruck, dann bei 0.001 Torr und maximal $70^{\circ} \mathrm{C}$ eingeengt. Der rotbraune Rückstand wird mit Ether aufgeschlämmt. Vom Unlöslichen wird abfiltriert, das Filtrat eingeengt und das zurückbleibende zähviskose schwarze Öl mittels Chromatographie über Kieselgel mit Petrolether/Ethylacetat (10:1) in 3 Fraktionen getrennt. 1. Fraktion: Ausb. 3.6 g (54\%) 2-Benzoyl-1methyl-4,5-bis(trimethylsilyl)imidazol (23a), zähviskoses gelbliches Öl.

$$
\begin{array}{ll}
\mathrm{C}_{17} \mathrm{H}_{26} \mathrm{~N}_{2} \mathrm{OSi}_{2} \text { (330.6) } & \text { Ber. C } 61.77 \text { H } 7.93 \text { N } 8.47 \\
& \text { Gef. C } 61.62 \mathrm{H} 7.87 \text { N } 8.41
\end{array}
$$

1. Fraktion: Ausb. $349 \mathrm{mg}$ (7\%) 2-Benzoyl-1-methyl-4-(trimethylsilyl) imidazol (20a), farblose Kristalle, Schmp. $68-69^{\circ} \mathrm{C},{ }^{1} \mathrm{H}-\mathrm{NMR}$ spektroskopisch identisch mit dem aus 11 a dargestellten 20 a. -3 . Fraktion: Ausb. $737 \mathrm{mg}$ (14\%) 2-Benzoyl-1-methyl-5-(trimethylsilyl) imidazol (14a), zähviskoses, gelbliches Ö1, 'H-NMR-spektroskopisch identisch mit dem aus 3 a dargestellten $14 a$.

d) Mit 4-Toluoylchlorid: Zu $575.2 \mathrm{mg}$ (2.54 mmol) 9 in $5 \mathrm{ml}$ Acetonitril werden unter Eiskühlung und Rühren innerhalb 9 min $393.0 \mathrm{mg}$ (2.54 mmol) 4-Toluoylchlorid, dann innerhalb $6 \mathrm{~min}$ $257.0 \mathrm{mg}$ ( $2.54 \mathrm{mmol})$ Triethylamin getropft. Es wird $40 \mathrm{~h}$ bei Raumtemp. gerührt, dann $1 \mathrm{ml}$ Methanol zugegeben, weitere $40 \mathrm{~h}$ gerührt, im Rotationsverdampfer bei maximal $40^{\circ} \mathrm{C}$ eingeengt, der braune Rückstand mit Ether suspendiert und abgesaugt. Das Filtrat wird eingeengt, das zurückbleibende zähviskose dunkelbraune Öl (684 mg) mit $4 \mathrm{ml}$ Pentan versetzt, und vom Unlöslichen wird abfiltriert. Nach 5-wöchigem Stehenlassen werden die gebildeten Kristalle mittels Chromatographie über Kieselgel mit Petrolether/ Ethylacetat (20:1) in 3 Fraktionen getrennt. 1. Fraktion: Nach weiterer chromatographischer Reinigung über Kieselgel mit Petrolether/Ethylacetat (20:1) Ausb. $277 \mathrm{mg}$ (32\%) 1-Methyl-2-(4-toluoyl)-4,5-bis (trimethylsilyl)imidazol (23c), Schmp. $120.5^{\circ} \mathrm{C}$.

$$
\begin{array}{ll}
\mathrm{C}_{18} \mathrm{H}_{28} \mathrm{~N}_{2} \mathrm{OSi}_{2} \text { (344.6) } & \text { Ber. C 62.74 H 8.18 N } 8.13 \\
& \text { Gef. C 62.82 H 8.24 N 8.11 }
\end{array}
$$

2. Fraktion: Ausb. $36 \mathrm{mg}$ (5\%) 1-Methyl-2-(4-toluoyl)-4-(trimethylsilyl)imidazol (20c), zähviskoses farbloses Öl. - ${ }^{1} \mathrm{H}-\mathrm{NMR}$ $\left(\mathrm{CDCl}_{3}\right): \delta=7.18$ (s, $\left.1 \mathrm{H}, 5-\mathrm{H}\right)$.

$$
\begin{array}{ll}
\mathrm{C}_{15} \mathrm{H}_{20} \mathrm{~N}_{2} \mathrm{OSi}(272.4) & \text { Ber. C } 66.13 \text { H } 7.40 \mathrm{~N} 10.28 \\
& \text { Gef. C } 65.97 \text { H } 7.42 \mathrm{~N} 10.11
\end{array}
$$

3. Fraktion: $139 \mathrm{mg}(20 \%)$ 1-Methyl-2-(4-toluoyl)-5-(trimethylsilyl)imidazol (14d), zähviskoses farbloses Öl. - ${ }^{1} \mathrm{H}-\mathrm{NMR}\left(\mathrm{CDCl}_{3}\right)$ : $\delta=7.25(\mathrm{~s}, 1 \mathrm{H}, 4-\mathrm{H})$.

$$
\begin{array}{ll}
\mathrm{C}_{15} \mathrm{H}_{20} \mathrm{~N}_{2} \mathrm{OSi} \text { (272.4) } & \text { Ber, C } 66.13 \text { H } 7.40 \text { N } 10.28 \\
& \text { Gef. C 65.94 H } 7.42 \text { N } 10.12
\end{array}
$$

2. Umsetzungen von 23b: a) Mit Benzaldehyd: Zu der Suspension von $25.8 \mathrm{mg}$ ( $0.17 \mathrm{mmol}) \mathrm{CsF}$ in $1.7 \mathrm{ml}$ Dimethylformamid werden bei Raumtemp. $377.0 \mathrm{mg}$ ( $3.55 \mathrm{mmol}$ ) Benzaldehyd gegeben, dann wird innerhalb $10 \mathrm{~min}$ die Lösung von $1.217 \mathrm{~g}$ (3.33 mmol) $23 \mathrm{~b}$ in $6 \mathrm{ml}$ Dimethylformamid zugetropft, $50 \mathrm{~min}$ gerührt, mit Ether versetzt, vom Unlöslichen abgesaugt und das Filtrat bei maximal $70^{\circ} \mathrm{C}$ zunächst bei 15 Torr, dann bei 0.001 Torr eingeengt. Der Rückstand (1.46 g zähviskoses gelbes Öl) wird mit $25 \mathrm{ml}$ Ethanol und $306.7 \mathrm{mg}$ (6.66 mmol) Ameisensäure $22 \mathrm{~h}$ bei Raumtemp. gerührt und das Gemisch im Rotationsverdampfer bei maximal $50^{\circ} \mathrm{C}$ eingeengt. Das zurückbleibende ölige Kristallgemisch wird in Ethylacetat aufgenommen, die Lösung mit $20 \mathrm{ml}$ ges. NatriumhydrogencarbonatLösung neutralisiert und viermal mit insgesamt $80 \mathrm{ml}$ Ethylacetat extrahiert. Die vereinigten Extrakte werden mit Natriumsulfat getrocknet und eingeengt. Der Rückstand (1.176 g gelbliche Kristalle, Schmp. $115-130^{\circ} \mathrm{C}$ ) wird mit $20 \mathrm{ml}$ Pentan suspendiert, abgesaugt und mit $5 \mathrm{ml}$ Pentan gewaschen, Ausb. $593 \mathrm{mg}$ (45\%) 2-(4-Chlorbenzoyl)-5-(hydroxyphenylmethyl)-1-methyl-4-(trimethylsilyl)imidazol (24a), Schmp. $146^{\circ} \mathrm{C}$.

$$
\begin{aligned}
& \mathrm{C}_{21} \mathrm{H}_{23} \mathrm{ClN}_{2} \mathrm{O}_{2} \mathrm{Si}(399.0) \\
& \text { Ber. C } 63.22 \mathrm{H} 5.81 \mathrm{Cl} 8.89 \text { N } 7.02 \\
& \text { Gef. C } 63.04 \text { H } 5.86 \mathrm{Cl} 9.09 \text { N } 6.91
\end{aligned}
$$

b) Mit Benzoylfluorid: $\mathrm{Zu}$ der Suspension von $0.304 \mathrm{~g}(2.0 \mathrm{mmol})$ $\mathrm{CsF}$ in $6 \mathrm{ml}$ Dimethylformamid werden $2.482 \mathrm{~g} \mathrm{(20.0 \textrm {mmol } ) \mathrm { Ben } -}$ zoylfluorid gegeben, dann wird innerhalb $1 \mathrm{~h}$ bei $40^{\circ} \mathrm{C}$ die Lösung von $3.650 \mathrm{~g}(10.0 \mathrm{mmol}) 23 \mathrm{~b}$ in $30 \mathrm{ml}$ Dimethylformamid zugetropft, $1 \mathrm{~h}$ bei $40^{\circ} \mathrm{C}$ gerührt, mit Ether versetzt, vom Unlöslichen abgesaugt und das Filtrat zunächst bei $40^{\circ} \mathrm{C}$ im Rotationsverdampfer, dann bei 0.001 Torr und maximal $55^{\circ} \mathrm{C}$ eingeengt. Der dunkelrote Rückstand wird über Kieselgel mit Ethylacetat/Petrolether (1:10) chromatographiert, das resultierende gelbrote ölige Kristallgemisch mit Ether digeriert (Ausb. $904 \mathrm{mg}$ farbloses Produkt, Schmp. $127.5-129^{\circ} \mathrm{C}$ ), die Mutterlauge eingeengt und der Rückstand mit Pentan digeriert (Ausb. $724 \mathrm{mg}$, Schmp. 129-130 ${ }^{\circ} \mathrm{C}$ ), Gesamtausb. $1.628 \mathrm{~g}(41 \%)$ 5-Benzoyl-2-(4-chlorbenzoyl)-1-methyl4-(trimethylsilyl)imidazol (24b).

$$
\begin{array}{ll}
\mathrm{C}_{21} \mathrm{H}_{21} \mathrm{ClN}_{2} \mathrm{O}_{2} \mathrm{Si} \text { (396.9) } & \text { Ber. C 63.54 H 5.33 N } 7.06 \\
& \text { Gef. C 63.30 H 5.38 N } 6.98
\end{array}
$$

Umsetzungen ausgehend von 1-(Dimethylsulfamoyl)imidazol (1 b) (zu Schema 5) 
a) 1 -( Dimethylsulfamoyl)-4-(trimethylsilyl)imidazol (11 b): Zu der Lösung von $10.51 \mathrm{~g}(60.0 \mathrm{mmol}) 1^{8,24)}$ in $230 \mathrm{ml}$ Dimethoxymethan werden nach Lit. ${ }^{8)}$ bei -18 bis $-13^{\circ} \mathrm{C}$ innerhalb 25 min $84.6 \mathrm{ml}$ (132.0 mmol) einer $1.6 \mathrm{~N}$ Butyllithium-Lösung in Hexan getropft, dann wird rasch auf $-5^{\circ} \mathrm{C}$ erwärmt, 25 min bei dieser Temp. gerührt und anschließend bei $-2^{\circ} \mathrm{C}$ innerhalb $25 \mathrm{~min}$ die Lösung von $17.93 \mathrm{~g}$ (165.0 mmol) TMSC in $20 \mathrm{ml}$ Dimethoxymethan zugetropft. Nach Erwärmen auf Raumtemp. innerhalb $1 \mathrm{~h}$ wird $21 \mathrm{~h}$ gerührt, dann das Produktgemisch aus $11 \mathrm{~b}$ und $1-$ ( $\mathrm{Di}$ methylsulfamoyl)-2,4-bis(trimethylsilyl)imidazol (25) (mittels GC bestimmt) mit Ether verdünnt und dreimal mit insgesamt $500 \mathrm{ml}$ Wasser gewaschen. Die vereinigten wäßrigen Phasen werden mit insgesamt $500 \mathrm{ml}$ Ether ausgeschüttelt, die vereinigten Etherextrakte mit Natriumsulfat getrocknet und bei maximal $60^{\circ} \mathrm{C}$ im Rotationsverdampfer eingeengt. Der braunschwarze Rückstand $(9.64 \mathrm{~g})$ wird über Kieselgel mit Ethylacetat/Petrolether (1:1) chromatographiert, Ausb. $2.79 \mathrm{~g} \mathrm{(19 \% )} 11 \mathrm{~b}$, Schmp. $96.5^{\circ} \mathrm{C} .-{ }^{1} \mathrm{H}-$ $\operatorname{NMR}\left(\mathrm{CDCl}_{3}\right): \delta=7.32(\mathrm{~s}, 1 \mathrm{H}, 5-\mathrm{H}), 8.05(\mathrm{~s}, 1 \mathrm{H}, 2-\mathrm{H})$.

\section{$\mathrm{C}_{8} \mathrm{H}_{17} \mathrm{~N}_{3} \mathrm{O}_{2} \mathrm{SSi}$ (247.4) \\ Ber. C 38.84 H 6.93 N $16.99 \mathrm{~S} 12.96$ \\ Gef. C 38.96 H 6.81 N 16.74 S 12.95}

b) 1-(Dimethylsulfamoyl)-5-(trimethylsilyl)imidazol (4b): $\mathrm{Zu}$ $10.51 \mathrm{~g}(60.0 \mathrm{mmol}) \mathbf{1 b}, 21.51 \mathrm{~g}(198.0 \mathrm{mmol})$ TMSC und $130 \mathrm{ml}$ Tetrahydrofuran werden bei $-70^{\circ} \mathrm{C}$ innerhalb $3 \mathrm{~h} 235 \mathrm{ml}(163.0$ mmol) einer $0.695 \mathrm{~N}$ Lithiumdiisopropylamid-Lösung in Tetrahydrofuran getropft, dann wird $30 \mathrm{~min}$ bei $-73^{\circ} \mathrm{C}$ gerührt, innerhalb $2 \mathrm{~h}$ auf Raumtemp. erwärmt und noch 135 min gerührt. Das Gemisch aus 1-(Dimethylsulfamoyl)-2,5-bis(trimethylsilyl)imidazol (3b) und $4 \mathrm{~b}$ (mittels GC bestimmt) wird mit $100 \mathrm{ml}$ absol. Methanol versetzt, nach $1 \mathrm{~h}$ im Rotationsverdampfer bei maximal $50^{\circ} \mathrm{C}$ eingeengt und der Rückstand mit jeweils $500 \mathrm{ml}$ Ether und Ethylacetat versetzt. Nach Erhitzen zum Sieden wird von Lithiumchlorid abfiltriert, das Filtrat im Rotationsverdampfer eingeengt, der Rückstand (21.4 g) mit 11 Ethylacetat/Hexan (3:1) erwärmt, vom Unlöslichen wird abfiltriert und das Filtrat im Rotationsverdampfer eingeengt. Der Rückstand (17.77 g) wird mit Ether versetzt, vom Unlöslichen wird abfiltriert, das Filtrat im Rotationsverdampfer eingeengt und der Rückstand (16.27 g) über Kieselgel mit Ethylacetat/Petrolether $(1: 2)$ chromatographiert, Ausb. $12.78 \mathrm{~g}(85 \%) \mathbf{4 b}$, Schmp. ca. $100^{\circ} \mathrm{C} .-{ }^{1} \mathrm{H}-\mathrm{NMR}\left(\mathrm{CDCl}_{3}\right): \delta=7.20(\mathrm{~s}, 1 \mathrm{H}, 4-\mathrm{H}), 8.06$ (s, $1 \mathrm{H}, 2-\mathrm{H})$.

\section{$\mathrm{C}_{8} \mathrm{H}_{17} \mathrm{~N}_{3} \mathrm{O}_{2} \mathrm{SSi}(247.4)$}

Ber. C 38.84 H 6.93 N 16.99 S 12.96

Gef. C 38.70 H 6.79 N 17.05 S 13.25

CsF-katalysierte Umsetzung von $\mathbf{4 b}$ mit Benzaldehyd (zu Schema 5) a) $\mathrm{Zu}$ der Suspension von $45.6 \mathrm{mg}(0.3 \mathrm{mmol}) \mathrm{CsF}$ und $1.17 \mathrm{~g}$ $(11.0 \mathrm{mmol})$ Benzaldehyd in $5 \mathrm{ml}$ Dimethylformamid wird bei $60^{\circ} \mathrm{C}$ innerhalb $10 \mathrm{~min}$ die Lösung von $2.47 \mathrm{~g}(10.0 \mathrm{mmol}) \mathbf{4 b}$ in $5 \mathrm{ml}$ Dimethylformamid getropft. Nach $35 \mathrm{~min}$. Rühren wird das Gemisch auf Raumtemp. abgekühlt, mit Ether versetzt und abgesaugt. Das Filtrat wird zunächst im Rotationsverdampfer, dann bei 0.005 Torr und $60^{\circ} \mathrm{C}$ eingeengt. Die öligen dunkelgelben Kristalle werden mehrmals mit Ether digeriert, es wird eingeengt, mit $100 \mathrm{ml}$ Hexan/ Ethylacetat/Ether $(90: 5: 5)$ versetzt, erwärmt und vom Unlöslichen abfiltriert. Das Filtrat wird auf $40 \mathrm{ml}$ eingeengt und mit $20 \mathrm{ml} \mathrm{Hep-}$ tan versctzt, die gebildeten Kristalle werden abgesaugt $(2.325 \mathrm{~g}$, Schmp. $74.5-76.5^{\circ} \mathrm{C}$ ). Die Mutterlauge wird im Rotationsverdampfer eingeengt und der Rückstand $(783 \mathrm{mg}$ ) aus Pentan umkristallisiert, Ausb. $383 \mathrm{mg}$, Schmp. $72-75.5^{\circ} \mathrm{C}$. Gesamtausb. $2.71 \mathrm{~g}$ (77\%) 1-(Dimethylsulfamoyl)-5-[phenyl(trimethylsiloxy)methyl]- imidazol (17b). - ${ }^{1} \mathrm{H}-\mathrm{NMR}\left(\mathrm{CDCl}_{3}\right): \delta=7.09(\mathrm{~s}, 1 \mathrm{H}, 4-\mathrm{H}), 7.88(\mathrm{~s}$, $1 \mathrm{H}, 2-\mathrm{H})$.

\author{
$\mathrm{C}_{15} \mathrm{H}_{23} \mathrm{~N}_{3} \mathrm{O}_{3} \mathrm{SSi}$ (353.5) \\ Ber. C 50.96 H $6.56 \mathrm{~N} 11.89$ S 9.07 \\ Gef. C 51.03 H 6.50 N 11.87 S 9.07
}

b) $\mathrm{Zu}$ der Suspension von $45.6 \mathrm{mg}(0.3 \mathrm{mmol}) \mathrm{CsF}$ und $1.17 \mathrm{~g}$ (11.0 mmol) Benzaldehyd in $5 \mathrm{ml}$ Dimethylformamid wird bei $60^{\circ} \mathrm{C}$ innerhalb $10 \mathrm{~min}$ die Lösung von $2.47 \mathrm{~g}(10.0 \mathrm{mmol}) \mathbf{4 b}$ in $5 \mathrm{ml}$ Dimethylformamid getropft. Nach 1stdg. Rühren bei $60^{\circ} \mathrm{C}$ werden $0.36 \mathrm{ml}(20.0 \mathrm{mmol})$ Wasser zugegeben, dann wird $40 \mathrm{~h}$ bei $60^{\circ} \mathrm{C}$ gerührt, auf Raumtemp. abgekühlt, das Gemisch in $30 \mathrm{ml}$ wäßrige Natriumhydrogencarbonat-Lösung gegeben und mit insgesamt $200 \mathrm{ml} \mathrm{Ethylacetat/Ether} \mathrm{(2:1)} \mathrm{ausgeschüttelt.} \mathrm{Die} \mathrm{vereinigten} \mathrm{or-}$ ganischen Phasen werden zweimal mit jeweils $30 \mathrm{ml}$ Wasser gewaschen, mit Natriumsulfat getrocknet und im Rotationsverdampfer eingeengt. Der farblose Rückstand wird mit Ethylacetat/Ether umkristallisiert, Ausb. $2.16 \mathrm{~g}(77 \%) 1$-(Dimethylsulfamoyl)-5-(hydroxyphenylmethyl)imidazol (17c), Schmp. $135.5^{\circ} \mathrm{C} .-{ }^{1} \mathrm{H}-\mathrm{NMR}$ $\left(\mathrm{CDCl}_{3}\right): \delta=6.73(\mathrm{~s}, 1 \mathrm{H}, 4-\mathrm{H}), 8.15(\mathrm{~s}, 1 \mathrm{H}, 2-\mathrm{H})$.

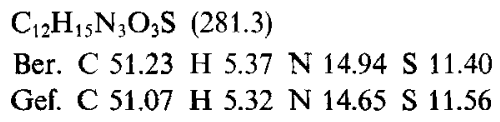

5-Benzoylimidazol (26): $\mathrm{Zu}$ der Lösung von $703 \mathrm{mg}$ (2.5 mmol) $17 \mathrm{c}$ in $10 \mathrm{ml}$ Eisessig wird bei Raumtemp. die Lösung von $9.82 \mathrm{mg}$ ( $3.3 \mathrm{mmol}$ ) $\mathrm{Na}_{2} \mathrm{Cr}_{2} \mathrm{O}_{7} \cdot 2 \mathrm{H}_{2} \mathrm{O}$ gegeben und $2.5 \mathrm{~h}$ unter Rückfluß erhitzt. Nach Abkühlen auf Raumtemp. wird das Gemisch mit wäßriger Natriumhydrogencarbonat-Lösung auf $\mathrm{pH} 11-12$ eingestellt, viermal mit jeweils $150 \mathrm{ml}$ Ether, viermal mit jeweils $50 \mathrm{ml}$ Dichlormethan, zweimal mit jeweils $40 \mathrm{ml}$ Ether und einmal mit $20 \mathrm{ml}$ Methanol extrahiert. Die vereinigten organischen Extrakte werden mit $20 \mathrm{ml}$ Wasser gewaschen und im Rotationsverdampfer zur Trockne eingeengt. Der Rückstand wird mit Ethylacetat/Ether digeriert, die gebildeten Kristalle werden mit Methanol aufgeschlämmt, abfiltriert und mit $50 \mathrm{ml}$ Petrolether/Ethylacetat/Aceton (2:2:1) versetzt. Der grüne Feststoff wird abfiltriert, das Filtrat im Rotationsverdampfer eingeengt und der farblose kristalline Rückstand $\left(200 \mathrm{mg}\right.$, Schmp. $\left.135-138^{\circ} \mathrm{C}\right)$ bei $110-115^{\circ} \mathrm{C} / 0.001$ Torr sublimiert, Ausb. $185 \mathrm{mg}(43 \%)$, Schmp. $140-144^{\circ} \mathrm{C}$ [Lit. ${ }^{5)} 142^{\circ} \mathrm{C}$ (Aceton/Hexan)]. $-{ }^{1} \mathrm{H}-\mathrm{NMR}\left(\mathrm{CDCl}_{3}\right): \delta=7.75$ (s, $\left.1 \mathrm{H}, 4(5)-\mathrm{H}\right)$, $7.77-8.03\left(\mathrm{~m}, 1 \mathrm{H}, 2-\mathrm{H}+2 \mathrm{H}, \mathrm{H}_{0} \mathrm{Ph}\right)$.

Umsetzungen ausgehend von 1-(Dimethylsulfamoyl) pyrazol (27 b) (zu Schema 6)

1-(Dimethylsulfamoyl)pyrazol (27b): Zu der Lösung von $34.04 \mathrm{~g}$ $(0.50 \mathrm{~mol})$ Pyrazol (27a) in $570 \mathrm{ml}$ Benzol werden bei Raumtemp. $46.55 \mathrm{~g}(0.46 \mathrm{~mol})$ Triethylamin gegeben, dann innerhalb $20 \mathrm{~min}$ $61.74 \mathrm{~g}(0.43 \mathrm{~mol})$ Dimethylsulfamoylchlorid ${ }^{25)}$ zugetropft. Nach Rühren $\left(1 \mathrm{~h}\right.$ bei $35^{\circ} \mathrm{C}, 21 \mathrm{~h}$ bei $60^{\circ} \mathrm{C}$ und $22 \mathrm{~h}$ unter Rückfluß) und Abkühlen wird Triethylammoniumchlorid $(65 \mathrm{~g}, 95 \%)$ abfiltriert, aus dem Filtrat zunächst im Rotationsverdampfer bei $60^{\circ} \mathrm{C}$ Benzol entfernt, dann das Produkt bei 0.001 Torr überdestilliert und anschließend bei 16 Torr destilliert, Ausb. $43.8 \mathrm{~g}(59 \%)$, Sdp. $118.5^{\circ} \mathrm{C}$ $-{ }^{1} \mathrm{H}-\mathrm{NMR}\left(\mathrm{CDCl}_{3}\right): \delta=6.38\left(\mathrm{t}, 1 \mathrm{H},{ }^{3} \mathrm{~J}=1 \mathrm{~Hz}, 4-\mathrm{H}\right), 7.69(\mathrm{~d}$, $1 \mathrm{H}, 3-\mathrm{H}), 7.95$ (d, $1 \mathrm{H}, J=2 \mathrm{~Hz}, 5-\mathrm{H})$

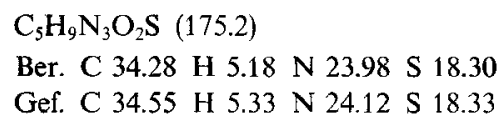

Abspaltung der Schutzgruppe in $27 \mathrm{~b}: 1.75 \mathrm{~g}(10.0 \mathrm{mmol}) 27 \mathrm{~b}$ werden in $250 \mathrm{ml}(50.0 \mathrm{mmol}) 2 \mathrm{~N} \mathrm{HCl} 21 \mathrm{~h}$ unter Rückfluß erhitzt. Nach Abkühlen wird das Gemisch mit $100 \mathrm{ml} 1 \mathrm{~N}$ Natriumcarbonat-Lösung auf pH 10 eingestellt und fünfmal mit insgesamt $200 \mathrm{ml}$ Ether ausgeschüttelt. Die vereinigten Extrakte werden mit Natriumsulfat getrocknet, der Ether wird im Rotationsverdampfer 
bei $30^{\circ} \mathrm{C}$ entfernt, Ausb. $430 \mathrm{mg}$ (ca. 62\%) Pyrazol (27a) (Reinheit nach $\mathrm{GC}=98 \%$ ).

1-(Dimethylsulfamoyl)-5-(trimethylsilyl)pyrazol (28): $\mathrm{Zu} 5.26 \mathrm{~g}$ $(30.0 \mathrm{mmol}) 27 \mathrm{~b}$ in $100 \mathrm{ml}$ Ether und $20 \mathrm{ml}$ Tetrahydrofuran werden bei $-73^{\circ} \mathrm{C}$ innerhalb $1 \mathrm{~h}$ unter Rühren $21.2 \mathrm{ml}(33.0 \mathrm{mmol})$ $1.6 \mathrm{~N}$ Butyllithium-Lösung in Hexan getropft, und es wird noch $1.5 \mathrm{~h}$ bei $-73^{\circ} \mathrm{C}$ gerührt. Dann wird zu dem Gemisch bei $-73^{\circ} \mathrm{C}$ innerhalb $75 \mathrm{~min}$ die Lösung von $3.59 \mathrm{~g}(33.0 \mathrm{mmol})$ TMSC in $50 \mathrm{ml}$ Tetrahydrofuran getropft. Nach Erwärmen auf Raumtemp. wird abfiltriert, das Filtrat bei $30^{\circ} \mathrm{C}$ im Rotationsverdampfer eingeengt und das zurückbleibende gelbe Öl $(8.12 \mathrm{~g})$ bei 0.001 Torr über eine 18-cm-Spiegelkolonne destilliert, Ausb. $5.79 \mathrm{~g}(78 \%)$, Sdp. $66^{\circ} \mathrm{C} / 0.001$ Torr. $-{ }^{1} \mathrm{H}-\mathrm{NMR}\left(\mathrm{CDCl}_{3}\right) ; \delta=6.51(\mathrm{~d}, J=2 \mathrm{~Hz}, 1 \mathrm{H}$, 4-H), $7.71(\mathrm{~d}, 1 \mathrm{H}, 3-\mathrm{H})$.

$$
\begin{aligned}
& \mathrm{C}_{8} \mathrm{H}_{17} \mathrm{~N}_{3} \mathrm{O}_{2} \mathrm{SSi}(247.4) \\
& \text { Ber. C } 38.84 \mathrm{H} 6.93 \mathrm{~N} 16.99 \mathrm{~S} 12.96 \\
& \text { Gef. C } 38.86 \mathrm{H} 6.97 \mathrm{~N} 16.82 \mathrm{~S} 12.89
\end{aligned}
$$

1-(Dimethylsulfamoyl) -5-[phenyl(trimethylsiloxy) methyl]pyrazol (29a): $\mathrm{Zu}$ der Suspension von $0.152 \mathrm{~g}(1.0 \mathrm{mmol}) \mathrm{CsF}$ in $5 \mathrm{ml}$ Dimethylformamid werden $1.142 \mathrm{~g}(10.76 \mathrm{mmol})$ Benzaldehyd gegeben, dann wird bei $100^{\circ} \mathrm{C}$ innerhalb 7 min die Lösung von $2.493 \mathrm{~g}(10.08 \mathrm{mmol}) 28 \mathrm{in} 5 \mathrm{ml}$ Dimethylformamid zugetropft und 23 min gerührt. Nach Abkühlen wird das Gemisch mit $10 \mathrm{ml}$ Ether versetzt, es wird abfiltriert, das Filtrat bei 16 Torr und maximal $60^{\circ} \mathrm{C}$ eingeengt und der rosafarbene Rückstand mehrmals mit Ether und Pentan aufgeschlämmt. Vom ausgefallenen Feststoff wird abfiltriert und das Filtrat über eine 10-cm-Spiegelkolonne bei 0.001 Torr destilliert, Ausb. 2.38 g (67\%), Sdp. $119-121^{\circ} \mathrm{C} / 0.001$ Torr. - ${ }^{\prime} \mathrm{H}-\mathrm{NMR}\left(\mathrm{CDCl}_{3}\right): \delta=6.28$ (s, 2H, CHO, 4-H), 7.59 (d, $J=$ $2 \mathrm{~Hz}, 1 \mathrm{H}, 3-\mathrm{H})$.

$$
\begin{aligned}
& \mathrm{C}_{15} \mathrm{H}_{23} \mathrm{~N}_{3} \mathrm{O}_{3} \mathrm{SSi}(353.5) \\
& \text { Ber. C } 50.96 \mathrm{H} 6.56 \mathrm{~N} 11.89 \mathrm{~S} 9.07 \\
& \text { Gef. C } 50.87 \mathrm{H} 6.60 \mathrm{~N} 12.27 \mathrm{~S} 9.36
\end{aligned}
$$

\section{1-(Dimethylsulfamoyl)-5-(hydroxyphenylmethyl)pyrazol (29b)}

a) Die Lösung von $0.950 \mathrm{~g}(2.69 \mathrm{mmol}) 29 \mathrm{a}$ in $4.8 \mathrm{ml}$ Ethanol wird mit $286.0 \mathrm{mg}$ ( $6.2 \mathrm{mmol}$ ) Ameisensäure versetzt, $6 \mathrm{~h}$ bei Raumtemp. gerührt und dann bei 16 Torr und maximal $60^{\circ} \mathrm{C}$ im Rotationsverdampfer eingeengt. Das zurückbleibende farblose $\mathrm{O} l$ $(805 \mathrm{mg})$ wird in Ether aufgenommen und die Lösung mit $20 \mathrm{ml}$ $0.5 \mathrm{~N}$ Natriumhydrogencarbonat-Lösung ausgeschüttelt. Die wäßrige Phase wird mehrmals mit insgesamt $80 \mathrm{ml}$ Ether ausgeschüttelt, die vereinigten Extrakte werden mit Magnesiumsulfat getrocknet und im Rotationsverdampfer eingeengt. Der Rückstand $(800 \mathrm{mg})$ wird über Kieselgel mit Petrolether/Ethylacetat $(1: 1)$ chromatographiert, Ausb. $573 \mathrm{mg}(76 \%)$, Schmp. $56-60{ }^{\circ} \mathrm{C} .-{ }^{1} \mathrm{H}-$ NMR $\left(\mathrm{CDCl}_{3}\right): \delta=6.04(\mathrm{~d}, J=2 \mathrm{~Hz}, 1 \mathrm{H}, 4-\mathrm{H}), 7.56(\mathrm{~d}, 1 \mathrm{H}$, $3-\mathrm{H})$.

$$
\begin{aligned}
& \mathrm{C}_{12} \mathrm{H}_{15} \mathrm{~N}_{3} \mathrm{O}_{3} \mathrm{~S}(281.3) \\
& \text { Ber. C } 51.23 \text { H } 5.37 \mathrm{~N} 14.94 \text { Si } 11.40 \\
& \text { Gef. C } 51.42 \text { H } 5.34 \text { N } 14.76 \text { Si } 11.45
\end{aligned}
$$

b) Bei $80^{\circ} \mathrm{C}$ werden zu der Suspension von $0.381 \mathrm{~g}(2.51 \mathrm{mmol})$ $\mathrm{CsF}$ in $25 \mathrm{ml}$ Dimethylformamid $5.59 \mathrm{~g}(52.7 \mathrm{mmol})$ Benzaldehyd gegeben, dann wird innerhalb $40 \mathrm{~min}$ die Lösung von $12.42 \mathrm{~g}(50.2$ mol) 28 in $25 \mathrm{ml}$ Dimethylformamid zugetropft. Nach $45 \mathrm{~min}$. Rühren bei $80^{\circ} \mathrm{C}$ wird $1 \mathrm{ml}$ Wasser, nach weiteren $20 \mathrm{~min}$ nochmals $1 \mathrm{ml}$ Wasser zugegeben und noch $15 \mathrm{~min}$ gerührt. Nach Abkühlen auf Raumtemp. wird das Gemisch in $200 \mathrm{ml}$ wäßrige Natriumhydrogencarbonat-Lösung eingerührt und mehrmals mit insgesamt 1.21 Ethylacetat/Ether (2:1) ausgeschüttelt. Die vereinigten organischen Extrakte werden zweimal mit jeweils $100 \mathrm{ml}$ Wasser gewaschen und im Rotationsverdampfer bei Raumtemp. zur Trockne eingeengt. Der Rückstand wird mehrmals mit Ether digeriert und die Lösung wieder eingeengt, bis Kristallisation erfolgt. Das hellgelbe Rohprodukt (14.4 g) wird über Kieselgel mit Petrolether/ Ethylacetat (5:1) chromatographiert und anschließend aus hochsiedendem Petrolether mit einem geringen Anteil an Ethylacetat oder Ether umkristallisiert, Ausb. $11.49 \mathrm{~g}(81 \%)$, Schmp. $63-64^{\circ} \mathrm{C}$, ${ }^{1} \mathrm{H}$-NMR-spektroskopisch identisch mit der nach a) dargestellten Verbindung.

5-(Hydroxyphenylmethyl)pyrazol (30a): $705.0 \mathrm{mg}(2.51 \mathrm{mmol})$ $29 \mathrm{~b}$ werden in $10 \mathrm{ml}$ Eisessig gelöst und $5 \mathrm{~h}$ bei Raumtemp., dann $16 \mathrm{~h}$ unter Rückfluß gerührt. Nach Abkühlen auf Raumtemp. wird das Gemisch mit wäßriger Natriumhydrogencarbonat-Lösung auf pH 9-10 eingestellt, sechsmal mit jeweils $30 \mathrm{ml}$ Ethylacetat und zweimal mit jeweils $30 \mathrm{ml}$ Dichlormethan ausgeschüttelt. Die vereinigten organischen Extrakte werden mit $10 \mathrm{ml}$ Wasser gewaschen und im Rotationsverdampfer eingeengt, Ausb. $336 \mathrm{mg}(77 \%)$, Schmp. $95-125^{\circ} \mathrm{C}$, nach Sublimation bei 0.001 Torr bis $100^{\circ} \mathrm{C}$ $243 \mathrm{mg}(56 \%)$, Schmp. $102-112^{\circ} \mathrm{C}$ [ Lit. $\left.^{26)} 114^{\circ} \mathrm{C}\left(\mathrm{CHCl}_{3}\right)\right],{ }^{1} \mathrm{H}-$ NMR-spektroskopisch identisch mit Lit. ${ }^{26)}$.

5-Benzoylpyrazol (30 b): Die Lösung von $705.5 \mathrm{mg}(2.51 \mathrm{mmol})$ 29b in $7 \mathrm{ml}$ Eisessig wird bei Raumtemp. mit einer Lösung von $745.0 \mathrm{mg}$ ( $2.50 \mathrm{mmol}) \mathrm{Na}_{2} \mathrm{Cr}_{2} \mathrm{O}_{7} \cdot 2 \mathrm{H}_{2} \mathrm{O}$ in $1.5 \mathrm{ml}$ Wasser versetzt und $1 \mathrm{~h}$ unter Rückfluß erhitzt. Nach Abkühlen auf Raumtemp. wird das grüne Gemisch in Eis/Wasser eingerührt, vorsichtig mit halbgesättigter Natriumhydrogencarbonat-Lösung auf $\mathrm{pH} \mathrm{8-9}$ eingestellt, dreimal mit jeweils $50 \mathrm{ml}$ Ether und zweimal mit jeweils $25 \mathrm{ml}$ Ether und Dichlormethan ausgeschüttelt. Nach Waschen der vereinigten organischen Extrakte mit $20 \mathrm{ml}$ Wasser wird im Rotationsverdampfer eingeengt, der Rückstand zweimal mit Ether digeriert, abfiltriert und der farblose Feststoff ( $463 \mathrm{mg}$, Schmp. $84-96^{\circ} \mathrm{C}$ ) aus hochsiedendem Petrolether/Ether (2:1) umkristallisiert, Ausb. $298 \mathrm{mg}\left(69 \%\right.$ ), Schmp. $95-98^{\circ} \mathrm{C} \mathrm{(Lit.}{ }^{27)} 95-96^{\circ} \mathrm{C}$ ).

\section{CAS-Registry-Nummern}

1 a: $616-47-7$ / 1 b: 78162-58-0 / 3a: 50694-52-5 / 3b: 133228-18-9 / 4a: $50694-57-0$ / 4b: 107398-50-5/5: 872-49-1 / 6: 1003-91-4 / 7: 1003-50-5 / 8: 133227-91-5 / 9: 133227-92-6 / 10: 4897-21-6/11a: 133227-93-7 / 11 b: 133228-16-7 / 12a: 133227-94-8/12b: 10636843-8 / 13a: 133227-98-2 / 13b: 106368-44-9 / 14a: 133227-95-9/ 14b: $133227-96-0$ / 14c: 133227-97-1 / 14d: 133228-13-4 / 15a: 133227-99-3 / 15b: 133228-00-9 / 15c: 133228-01-0 / 16a: 13322802-1 / 16b: 133228-03-2 / 17a: 24132-86-3 / 17b: 133228-19-0/17c: 133228-20-3 / 18: 133228-04-3 / 19: 133270-00-5 / 20a: 13322805-4/20b: 133228-07-6 / 20c: 133228-12-3/21 : 133228-06-5/22a : 133228-08-7 / 22 b: 133228-25-8 / 23a: 133228-10-1/ 23b: 13322809-8 / 23c: $133228-11-2$ / 24a: $133228-14-5$ / 24b: 133228-15-6/ 25: 133228-17-8 / 26: 61985-32-8/ 27 a: 288-13-1/27b: 13322821-4 / 28: 133228-22-5 / 29 a: 133228-23-6 / 29 b: 133228-24-7/30a 19854-91-2 / 30 b: 19854-92-3

${ }^{1)}$ Herrn Professor Dr. Dr. h. c. Ekkehard Fluck zum 60. Geburtstag gewidmet. - Zur elektrophilen Aromatensubstitution, 37. Mitteilung. - 36. Mitteilung: F. Effenberger, J. König, Tetrahedron 44 (1988) 3281.

2) 2a) M. Roos, Dissertation, Univ. Stuttgart, 1986. - ${ }^{2 b)}$ R. Ahmad, A. v. Humboldt-Stipendiatin, Universität Stuttgart 1982 . 2c) R. Ahmad, J. Chem. Soc. Pak. 8 (1986) 175 [Chem. Abstr. 107 (1987) 77695r]; dort wurden einige unvollständig bearbeitctc Ergebnisse veröffentlicht.

3) 3a) M. R. Grimmett in Adv. Heterocycl. Chem. 27 (1980) 241. 3b) M. R. Grimmett in Comprehensive Heterocyclic Chemistry (K T. Potts, Ed.) Bd. 5, S. 457 ff, Pergamon Press, Oxford, New York, Toronto, Sydney, Paris, Frankfurt 1984. $-{ }^{3 \mathrm{c})}$ H. Schubert, J. Prakt. Chem. 8 (1959) 333. - ${ }^{3 d)}$ K. Gewald, G. Reinhold, Monatsh. Chem. 107 (1976) 1413. - ${ }^{3 e)}$ N. K. Kochetkov, I. Ambrush, T. I. Ambrush, Zh. Obshch. Khim. 29 (1959) 2964; J. Gen. Chem. USSR 29 (1959) 2927. - ${ }^{30}$ L. A. Reiter, J. Org. Chem. 49 (1984) 3494 
4) 4a) BASF A.-G. (H. Lermer, W. Hoelderich, T. Dockner, H. Koehler, Erf.), Eur. Pat. Appl. EP 300,32 (25. Jan. 1989), DE Appl. 3,724,035 (21. Juli 1987) [Chem. Abstr. 111 (1989) 23516x]. - 4b E. Regel, K.-H. Büchel, Liebigs Ann. Chem. 1977, 145. - ${ }^{4 c)}$ I. I. Grandberg, L. G. Vasina, A. S. Volkova, A. N. Kost, Zh. Obshch. Khim. 31 (1961) 1887; J. Gen. Chem. USSR 31 (1961) 1765. 4d) K. v. Auwers, H. Mauss, J. Prakt. Chem. 117 (1927) 311. - ${ }^{4 e)}$ L. Balbiano, G. Marchei, Gazz. Chim. Ital. 24 I (1894) 8. ${ }^{4 n}$ Sankyo Co., Ltd. Jpn. Tokkyo Koho JP 6004,821 [8504,821] (6. Feb. 1985) [Chem. Abstr. 103 (1985) 22 585s]

5) S. Iwasaky, Helv. Chim. Acta 59 (1976) 2738.

6) Kurkela (Farmos-Yhtyma Oy) (A.-J. Karjalaynen, K. O. A. Kurkela, Erf.), Eur. Pat. Appl. 24,829 (11. März 1981) [Chem. Abstr. 95 (1981) $115545 \mathrm{k}]$.

n) 7a) B. A. Tertov, Yu. V. Koshchienko, V. V. Bessonov, I. V. Kotenko (Rostov State University), U. S. S. R. SU 941,364 (7. Juli 1982), Appl. 2,930,627 (29. Mai 1980) [Chem. Abstr. 98 (1983) $53890 \mathrm{v}$ ]. - ${ }^{76)}$ B. A. Tertov, Yu. V. Koshchienko, V. V. Bessonov, Khim. Geterotsikl. Soedin 9 (1982) 1279 [Chem. Abstr. 98 (1983) 4587t]. - ${ }^{7 c)}$ R. Breslow, J. T. Hunt, R. Smiley, T. Tarnowski, J. Am. Chem. Soc. 105 (1983) 5337.

${ }^{8)}$ D. J. Chadwick, R. I. Ngochindo, J. Chem. Soc., Perkin Trans. $1,1984,481$.

9) 9a) B. Iddon, Heterocycles 23 (1985) 417. - ${ }^{9 b)}$ B. Iddon, Stud. Org. Chem. (Amsterdam) 1988, 35 [Chem. Abstr. 110 (1989) $114703 \mathrm{~b}]$, , a review with 61 references on the preparation and reactions of organolithium derivatives of azoles of synthetic importance". - ${ }^{90}$ B. Iddon, B. L. Lim, J. Chem. Soc., Perkin Trans. $1,1983,279$

${ }^{10}$ A. R. Katritzky, J. N. Lam, S. Sangupta, G. W. Rewcastle, Prog. Heterocycl. Chem. 1 (1989) 1.
11) B. J. Wakefield (Ed.), The Chemistry of Organolithium Compounds, Pergamon Press, Oxford, New York, Toronto, Sydney, Braunschweig 1974.

12) 12a) F. H. Pinkerton, S. F. Thames, J. Heterocycl. Chem. 6 (1969) 433. - i2b) P. Jutzi, H. J. Hoffmann, Chem. Ber. 106 (1973) 594

13) 13a) F. Effenberger, W. Spiegler, Chem. Ber. 118 (1985) 3872. 13b) F. Effenberger, W. Spiegler, Chem. Ber. 118 (1985) 3900.

14) F. Effenberger, A. Krebs, J. Org. Chem. 49 (1984) 4687.

15) 15a) P. Jutzi, W. SakriB, Chem. Ber. 106 (1973) 2815. - isb) F. H. Pinkerton, S. F. Thames, J. Heterocycl. Chem. 9 (1972) 67. ${ }^{15 c)}$ T. Ogawa, M. Yasui und M. Matsui, Agr. Biol. Chem. 36 (1972) 1441.

16) 16a) N. Gjos, S. Gronowitz, Acta Chem. Scand. 25 (1971) 2596. ${ }_{16 b)}$ M. D. Rausch, A. J. Sarnelli, Adv. Chem. Ser. 130 (1974) 240. - ${ }^{16 c)}$ J. Klein, S. Glily, D. Kast, J. Org. Chem. 35 (1970) 1280.

17) 17a) I. E. Balaban, F. L. Pyman, J. Chem. Soc. [London] 125 (1924) 1564 - ${ }^{17 b)}$ K. E. Stensiö, K. Wahlberg, R. Wahren, Acta Chem. Scand. 27 (1973) 2179.

18) B. E. Boulton, B. A. W. Coller, Aust. J. Chem. 27 (1974) 2331.

19) 19a) F. Effenberger, D. Häbich, Liebigs Ann. Chem. 1979, 842. 19b) D. Häbich, F. Effenberger, Synthesis 1979, 841.

${ }^{20)}$ D. S. Noyce, G. T. Stowe, J. Org. Chem. 38 (1973) 3762.

21) 21a) H. C. Kohn, Dissertation, Pennsylvania State University 1971 [Diss. Abstr. Int. BA 33 (1972) 89]. - ${ }^{21 b)}$ Y. Takeuchi, K. L. Kirk, L. A. Cohen, J. Org. Chem. 43 (1978) 3570.

22) J. A. Zoltewitz, L. S. Helmick, J. Am. Chem. Soc. 92 (1970) 7547.

${ }^{23)}$ K. L. Kirk, J. Heterocycl. Chem. 22 (1980) 57.

24) J. F. King, T. M. Lee, Can. J. Chem. 59 (1981) 356.

25) R. Heap, B. C. Saunders, J. Chem. Soc 1948, 1313.

26) C. Sabaté-Alduy, J. Bastide, Bull. Soc. Chim. Fr. 1972, 1764.

${ }^{27)}$ R. Gompper, R. Sobotta, Synthesis 1979, 385.

$[422 / 90]$ 